\title{
Integrated metabonomic-proteomic analysis reveals the effect of glucose stress on metabolic adaptation of Lactococcus lactis ssp. lactis CICC23200
}

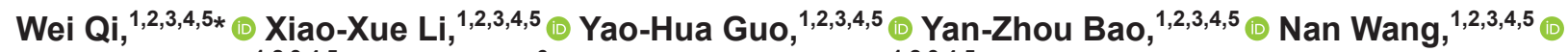 \\ Xue-Gang Luo, ${ }^{1,2,3,4,5}$ Chun-Di Yu, ${ }^{6}$ and Tong-Cun Zhang ${ }^{1,2,3,4,5 *}$ \\ ${ }^{1}$ State Key Laboratory of Food Nutrition and Safety, Tianjin University of Science \& Technology, Tianjin 300457, P.R. China \\ ${ }^{2}$ Key Laboratory of Industrial Fermentation Microbiology, Tianjin University of Science \& Technology, Ministry of Education, Tianjin 300457, \\ P.R. China \\ ${ }^{3}$ National Engineering Laboratory for Industrial Enzymes, Tianjin University of Science \& Technology, Tianjin 300457, P.R. China \\ ${ }^{4}$ Tianjin Key Laboratory of Industrial Microbiology, Tianjin University of Science \& Technology, Tianjin 300457, P.R. China \\ ${ }^{5}$ College of Biotechnology, Tianjin University of Science \& Technology, Tianjin 300457, P.R. China \\ ${ }^{6}$ College of Food Science and Engineering, Qingdao Agricultural University, Qingdao 266109, P.R. China
}

\begin{abstract}
A combined proteomic and metabonomic approach was used to investigate the metabolism of Lactococcus lactis ssp. lactis subjected to glucose stress treatment. A proteomic method was used to determine 1,427 altered proteins, including 278 proteins with increased expression and 255 proteins with decreased expression. A metabonomic approach was adopted to identify 98 altered metabolites, including 62 metabolites with increased expression and 26 metabolites with decreased expression. The integrated analysis indicated that the RNA and DNA mismatch repair process and energy metabolism were enhanced in response to high-glucose stress in L. lactis. Lactococcus lactis responded to glucose stress by up-regulating oxidoreductase activity, which acted on glycosyl bonds, hydrolase activity, and organic acid transmembrane transporter activity. This led to an improvement in the metabolic flux from glucose to pyruvate, lactate, acetate, and maltose. Downregulation of amino acid transmembrane transporter, aminoacyl-transfer RNA ligase, hydroxymethyl-, formyl-, and related transferase activities resulted in a decrease in the nitrogen metabolism-associated metabolic pathway, which might be related to inhibition of the production of biogenic amines. Overall, we highlight the response of metabolism to glucose stress and provide potential possibilities for the reduced formation of biogenic amines in improved level of sugar in the dairy fermentation industry. Moreover, according to the demand for industrial production, sugar concentration in
\end{abstract}

Received October 29, 2019.

Accepted April 14, 2020.

*Corresponding authors: qiweiwei@tust.edu.cn and tony@tust.edu .cn fermented foods should be higher, or lower, than a set value that is dependent on bacterial strain and biogenic amine yield.

Key words: Lactococcus lactis, glucose stress, proteomic, metabonomic

\section{INTRODUCTION}

Lactococcus lactis strains are widely used in food preservation and the dairy fermentation industry, especially in fermented milk products such as yogurt, kefir, and cheese, and are economically considered the most important lactic acid bacteria (Ruggirello et al., 2018). Moreover, L. lactis is considered a traditional food fermentation microorganism, and it is recognized as having generally regarded as safe (GRAS) status. Based on industrial interests, the L. lactis group includes 3 phenotypes: lactis (L. lactis ssp. lactis), cremoris ( $L$. lactis ssp. cremoris), and diacetylactis (L. lactis ssp. hordniae) phenotypes (Torres Manno et al., 2018). Lactococcus lactis has been characterized as having probiotic properties (Darby et al., 2019). Lactococcus lactis ssp. lactis can adapt easily to environments that contain milk or dairy products, and hence quickly and easily metabolize monosaccharides (glucose) and disaccharides (lactose; Yerlikaya, 2019).

During the initiation of the culture and industrial fermentation process, L. lactis encounters various environmental stress factors. Several studies have focused on the molecular adaptation mechanisms of L. lactis to various stresses such as acids (Tian et al., 2019), cadmium (Sheng et al., 2016), ethanol (Díez et al., 2017), salt (Gonzalez et al., 2017), and nitrogen sources (del Rio et al., 2015b). Recent results have shown that higher levels of up to $1 \mathrm{~g}$ per $100 \mathrm{~g}$ of glucose or lactose can reduce the yield of putrescine by repressing gene transcription involved in the agmatine deiminase path- 
way in L. lactis ssp. lactis and L. lactis ssp. cremoris (Linares et al., 2013; del Rio et al., 2015a). The strains involved in previous studies (Linares et al., 2013; del Rio et al., 2015a) showed that $10 \mathrm{~g} / \mathrm{L}$ of glucose or lactose concentration can decrease the yield of putrescine, indicating the sugar content in fermented dairy food should be higher than 1\%. Therefore, improving the glucose concentration might be a potential way to reduce the toxicity of substances such as biogenic amines in fermented foods and beverages. Moreover, according to the demand imposed by industrial production, the sugar concentration in fermented food should be higher than a set value or not lower than a set value depending on strain and biogenic amine yield: however, the molecular mechanisms underlying the proteomic and metabolic changes remain unclear. Furthermore, in the industrial fermentation environment, L. lactis might encounter local high sugar contents. In this study, proteomics analysis was performed by tandem mass tag (TMT) labeling technology combined with liquid chromatography-tandem mass spectrometry (LC-MS/ MS). A metabonomic analysis in L. lactis ssp. lactis was carried out after treatment in a glucose stress environment. The results will advance our understanding of the mechanisms underlying the metabolic adaptation of L. lactis used in fermented foods and beverages to higher levels of glucose and aim at establishing guidelines around the use of biogenic amine-producing strains and their proper application in the fermentation.

\section{MATERIALS AND METHODS}

\section{Lactic Acid Bacterial Strain and Growth Conditions}

The L. lactis ssp. lactis CICC 23200 used in this study was obtained from China Center of Industrial Culture Collection. Lactococcus lactis 23200 was first grown in $10 \mathrm{~mL}$ of M17 medium overnight, diluted in $100 \mathrm{~mL}$ of M17 medium, and inoculated during the logarithmic phase (optical density at $600 \mathrm{~nm} \approx 1.0$ ). For glucose treatments, $10 \mathrm{~mL}$ of cells were transferred into a $250-\mathrm{mL}$ anaerobic bottle up to the final volume of $250 \mathrm{~mL}$ medium containing 5, 10, 15, 30, 60, and 120 $\mathrm{m} M$ glucose, respectively. Next, the cells were grown at $30^{\circ} \mathrm{C}$ for $6 \mathrm{~h}$ and then collected for further analysis.

\section{Protein Extraction and Trypsin Digestion}

For protein extractions, samples were lysed ( $8 M$ urea, $1 \%$ protease inhibitor cocktail, sonication treatment). The supernatant was collected by centrifugation $\left(12,000 \times g, 4^{\circ} \mathrm{C}, 10 \mathrm{~min}\right)$ and the protein concentration was detected using a BCA kit. Protein digestion was conducted according to the method described in the previous work (Huang et al., 2018).

\section{TMT Labeling}

After trypsin digestion, peptides were labeled with TMT reagents in acetonitrile according to the TMT kit manufacturer's protocol (Thermo Fisher Scientific, Waltham, MA; https://www.thermofisher.com/order/ catalog/product $/ 90406 ?$ SID $=$ srch-srp-90406\#/90406 ?SID=srch-srp-90406). The peptides were then desalted using a Strata X C18 SPE column (Phenomenex, Torrance, $\mathrm{CA}$ ) and vacuum-dried.

\section{LC-MS/MS Analysis}

The LC-MS/MS analysis was conducted using MS/ MS in Q Exactive Plus (Thermo Fisher Scientific) coupled online to the EASY-nLC 1000 UPLC system (Thermo Fisher Scientific) with a 300Extend-C18 column (5 $\mathrm{m}$ particles, $4.6 \mathrm{~mm}$ i.d., $250 \mathrm{~mm}$ length, Agilent Technologies, Santa Clara, CA). The labeled peptides were first separated into 60 fractions (a gradient of $8 \%$ to $32 \%$ acetonitrile, $\mathrm{pH} 9.0,60 \mathrm{~min}$ ). Then, they were combined into 18 fractions and dried by vacuum centrifugation. The applied electrospray voltage was $2.0 \mathrm{kV}$, and the scan range was set to a full scan ranging from 350 to $1,800 \mathrm{~m} / z$. Labeled peptides were detected in the Orbitrap (Thermo Fisher Scientific) at a resolution of 70,000 and selected for MS/MS using an normalized collision energy setting of 28. Fragments were detected in the Orbitrap at a resolution of 17,500. A data-dependent procedure was applied that alternated between one MS scan followed by $20 \mathrm{MS} / \mathrm{MS}$ scans with $15.0 \mathrm{~s}$ dynamic exclusion. The automatic gain control was set at $5 \mathrm{E} 4$. The fixed first mass was set at $100 \mathrm{~m} / z$.

\section{Statistical Analysis}

The obtained MS/MS data were processed using the MaxQuant search engine (v.1.5.2.8; https://www .maxquant.org/). Tandem mass spectra were searched against the MASCOT 2.2 server database (www .matrixscience.com/) concatenated to the reverse decoy database. Protein sequences, Gene Ontology (GO), and Kyoto Encyclopedia of Genes and Genomes (KEGG) pathways were analyzed using the UniProt protein database (https://www.uniprot.org/) and KEGG database (https://www.kegg.jp/). The GO and KEGG enrichment analysis was undertaken using the hypergeometric test method with $\mathrm{R}$ language (false dis- 
covery rate $<1 \%$ and peptide minimum score $>40$ were considered significant).

\section{Metabolite Extraction and GC-MS Analysis}

Metabolite extraction and GC-MS analyses were performed using the method previously described (Qi et al., 2014). Metabolites were extracted in pure methanol and investigated by GC-MS after oximation and silylation derivatization. The GC-MS analyses were conducted with a Varian 4000 (Varian, Palo Alto, CA) GC/MS coupled to an HP 5973 quadrupole mass selective detector (Agilent Technologies) with an electron ionization source operated at $70 \mathrm{eV}$. The capillary column used for all analyses was a VF-5MS column (Agilent Technologies; $30 \times 0.25 \mu \mathrm{m} \times 0.25 \mathrm{~mm}$ ). Each compound was identified by reference to the NIST05a library (www.nist.gov/). Principal component analysis (PCA) was conducted with SPSS Statistics 18.0 package software (SPSS Inc., Chicago, IL). Metabolic pathways were investigated based on the KEGG database.

\section{RESULTS AND DISCUSSION}

\section{Effect of Glucose Treatment on Growth of $L$. lactis and $\mathrm{pH}$}

Growth of L. lactis and the $\mathrm{pH}$ of the medium exposed to glucose stress were detected (Figures 1A1B). The results showed a significant increase in the biomass of L. lactis with increasing glucose; however, the growth of L. lactis was slightly inhibited when the content of glucose reached $120 \mathrm{mM}$. Lactococcus lactis entered the stationary phase at $6 \mathrm{~h}$. The growth of $L$. lactis led to the acidification of the medium from $\mathrm{pH}$ 6.7 to approximately $\mathrm{pH} 6.2$ after $6 \mathrm{~h}$ of fermentation in cultures supplemented with $30 \mathrm{mM}$. Changes from $\mathrm{pH} 6.5$ to approximately $\mathrm{pH} 4.5$ occurred in 60 and 120 $\mathrm{m} M$ glucose. Therefore, through the combined analysis of growth and $\mathrm{pH}$, the metabonomic-proteomic analysis samples were obtained at $6 \mathrm{~h}$.

\section{Identification of Proteins Using TMT Labeling Technology Combined with LC-MS/MS}

The TMT correlation between the biological replicates was analyzed and the results are illustrated in Figure 1C. A total of 1,427 proteins were identified using TMT labeling technology combined with LC-MS/ MS, and the protein mass in the majority of the altered proteins ranged from 20 to $100 \mathrm{kDa}$ (Figure 1D). A protein was considered to be significantly expressed if it indicated at least a 1.2-fold change. Therefore, 278 proteins were up-regulated and 255 proteins were down-regulated in our data (Supplemental Table S1; https://doi.org/10.3168/jds.2019-17810). The subcellular location of the altered proteins was classified as cytoplasmic $(72 \%)$, membrane $(20 \%)$, and extracellular $(8 \%)$, respectively (Figure $1 \mathrm{E}$ ). The considerable proportion of down-regulated membrane proteins $(26 \%$ down-regulated proteins, 67 down-regulated proteins involved; $15 \%$ up-regulated proteins, 41 up-regulated proteins involved) in response to glucose stress indicated that these essential metabolic enzymes may be involved in the decrease of some metabolic pathways.

\section{Functional Classification and Annotation Analysis of Differentially Expressed Proteins}

Differentially expressed proteins of $L$. lactis were functionally classified by GO analysis, as shown in Figure 2. Differentially expressed proteins related to molecular functions were classified as follows: catalytic activity $(55 \%)$, binding (33\%), transporter activity $(6 \%)$, nucleic acid binding transcription factor activity $(2 \%)$, structural molecule activity (2\%), antioxidant activity, electron carrier activity, molecular transducer activity, and signal transducer activity (2\% each; Figure $2 \mathrm{~A})$.

Expressed proteins in L. lactis related to biological processes were classified as follows: metabolic processes (33\%), cellular processes (26\%), single-organism processes $(23 \%)$, localization (8\%), biological regulation $(5 \%)$, response to stimulus $(3 \%)$, cellular component organization or biogenesis (1\%), and signaling and biological adhesion (1\%; Figure 2B). As differentially expressed proteins focused on catalytic activity, metabolic processes are the most likely adaptable strategies to relieve the pressure of glucose stress.

\section{Functional Enrichment Analysis of Differentially Expressed Proteins}

Gene Ontology enrichment of differentially expressed proteins of $L$. lactis treated with glucose stress is demonstrated in Figure 3. Differentially expressed proteins related to molecular functions were enriched as follows: hydrolase activity, acting on glycosyl bonds, hydrolase activity, hydrolyzing O-glycosyl compounds, amino acid transmembrane transporter activity, hydroxymethyl-, formyl- and related transferase activity, oxidoreductase activity, acting on the $\mathrm{CH}-\mathrm{OH}$ group of donors, pyridoxal phosphate binding, and organic acid transmembrane transporter activity (Figure 3A). Differentially expressed proteins related to biological process were enriched as follows: pyrimidine-containing compound metabolic process, de novo inosine monophosphate (IMP) biosynthetic process, IMP metabolic process, IMP biosynthetic process, nucleobase metabolic pro- 

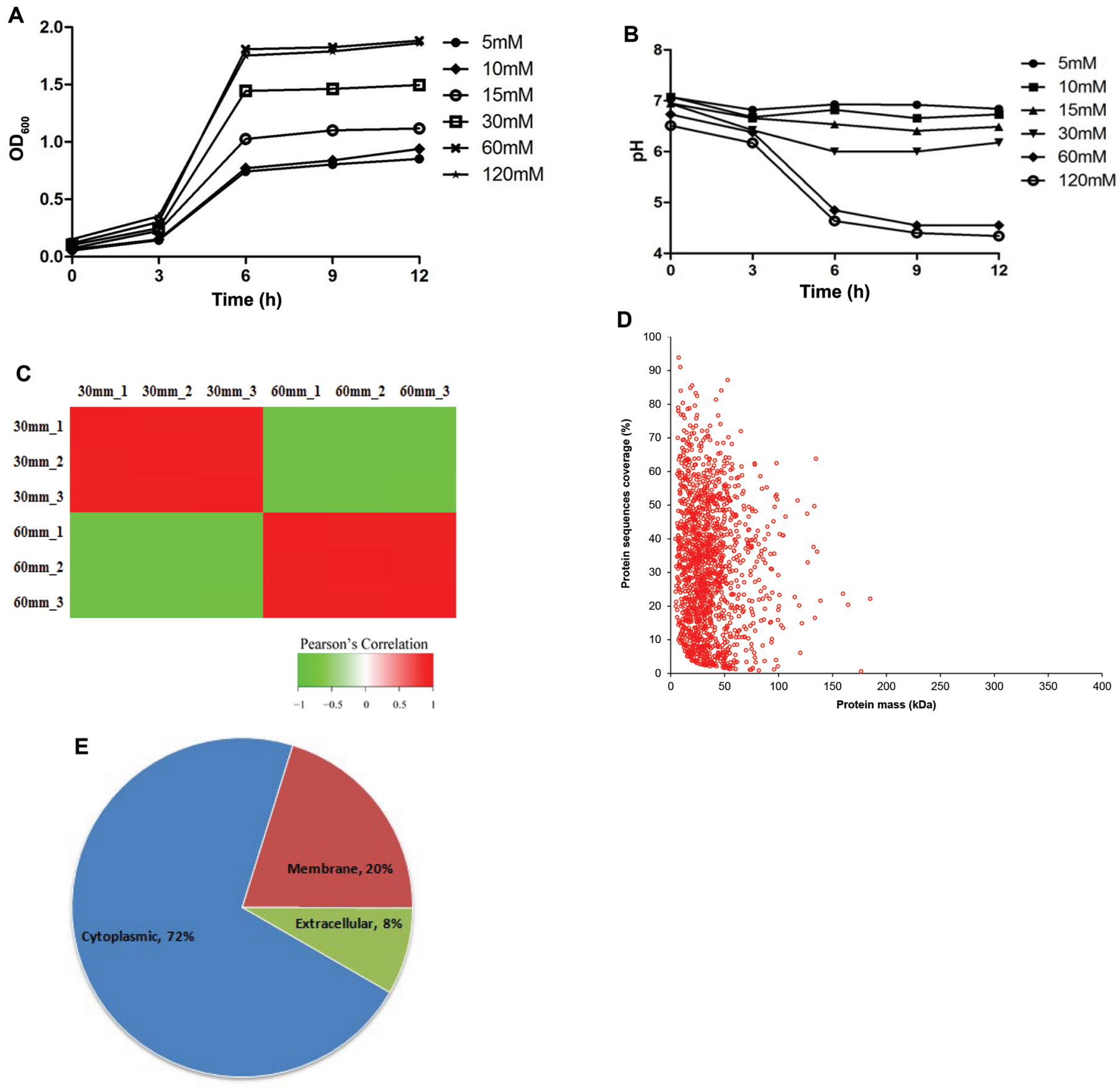

Figure 1. (A) The growth curves of Lactococcus lactis after treatment with 5, 10, 15, 30, 60, and $120 \mathrm{~m} M$ glucose; (B) $\mathrm{pH}$ of medium exposed to different glucose stresses; (C) Pearson's $\mathrm{R}^{2}$ showing the correlation of the differentially expressed proteins ( $\log _{2}$ ratio) between 3 biological replicates; (D) protein sequence coverage (\%); and (E) subcellular location of differentially expressed proteins in L. lactis. $\mathrm{OD}_{600}=\mathrm{optical}_{\text {den- }}$ sity at $600 \mathrm{~nm}$.

cess, pyrimidine-containing compound biosynthetic process, nitrogen compound transport, amide transport, peptide transport, and organophosphate catabolic process (Figure 3A).

Protein domains are high-level parts of proteins that exhibit evolutionary conservation, and many protein domains perform the specific functions of their proteins (Forslund et al., 2019). Differentially expressed proteins related to protein domains were enriched as follows: the amino acid permease/SLC12A domain, glycoside hydrolase, catalytic domain, oligopeptide/dipeptide ABC transporter, C-terminal, 6-phosphogluconate 

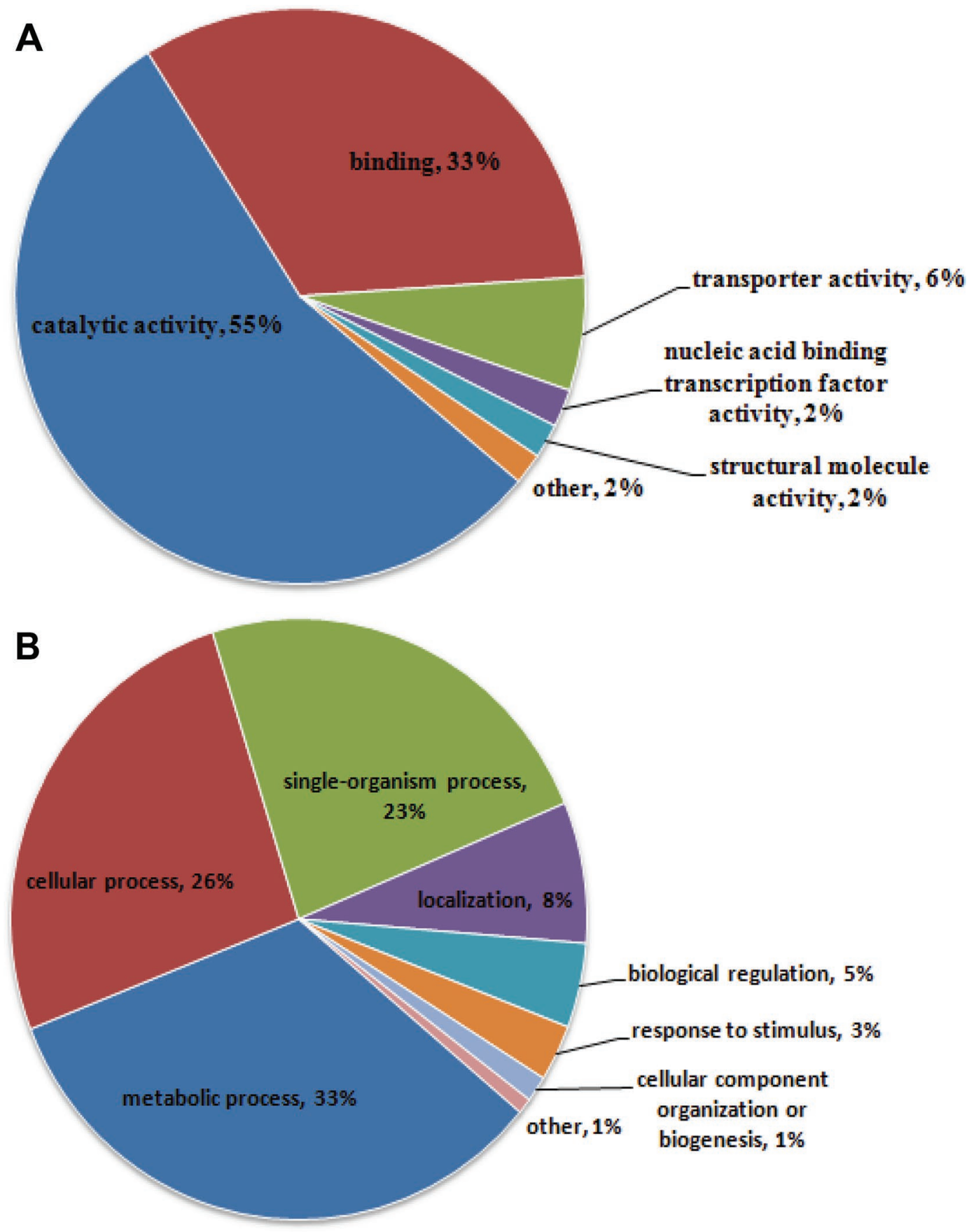

Figure 2. Gene Ontology categories for differentially expressed proteins and characteristics of Lactococcus lactis treated with glucose stress. (A) Functional classification of differentially expressed proteins in the molecular functions in response to glucose treatment, and (B) functional classification of differentially expressed proteins in the biological processes in response to glucose treatment.

dehydrogenase, domain 2, aminotransferase, class I/ class II, glycoside hydrolase superfamily, 6-phosphogluconate dehydrogenase, NADP-binding, biotin/lipoyl attachment, single hybrid motif, nitroreductase-like, dihydrofolate reductase-like domain, nitroreductase, and transfer RNA (tRNA)-binding arm (Figure 3B).
The KEGG pathways of differentially expressed proteins were analyzed (Figures 3C-3D). As shown in the figure, carbon metabolism (starch and sucrose metabolism, pyruvate metabolism, citrate cycle (tricarboxylic acid) and glycolysis/gluconeogenesis) could be enriched with increased protein expression (Figure 3C). Purine 
A

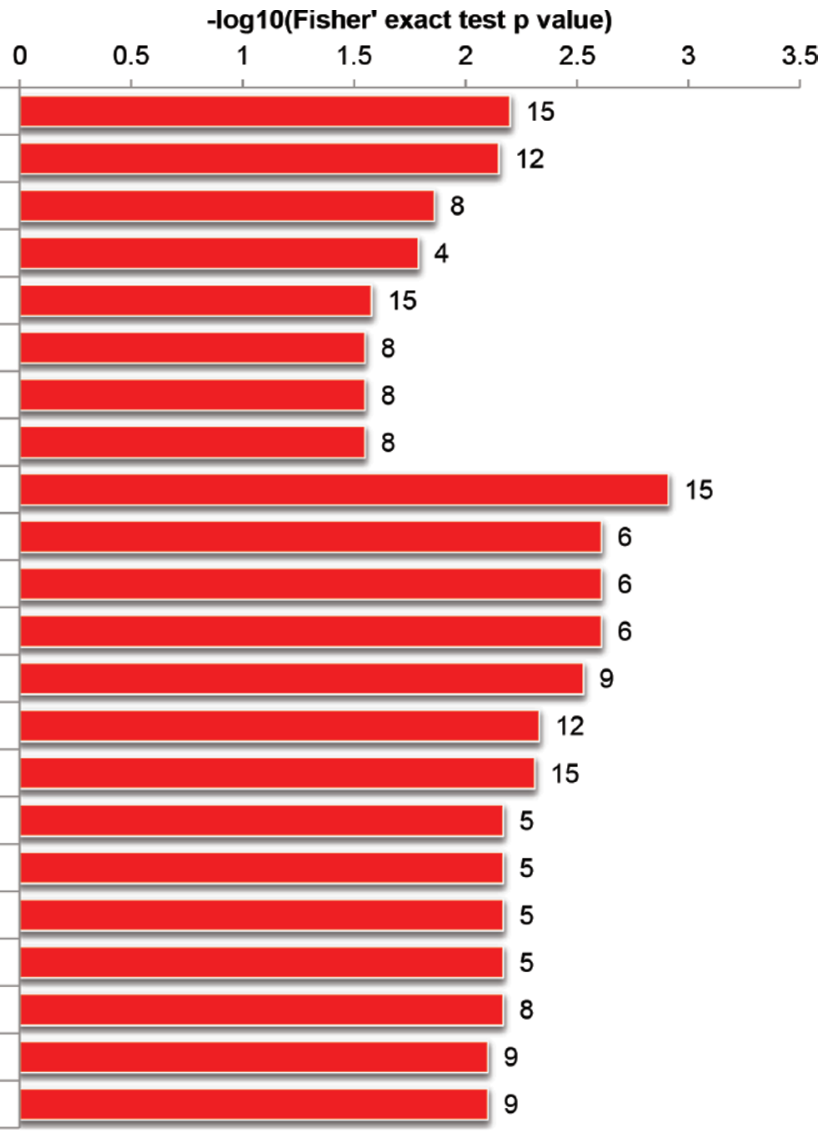

B

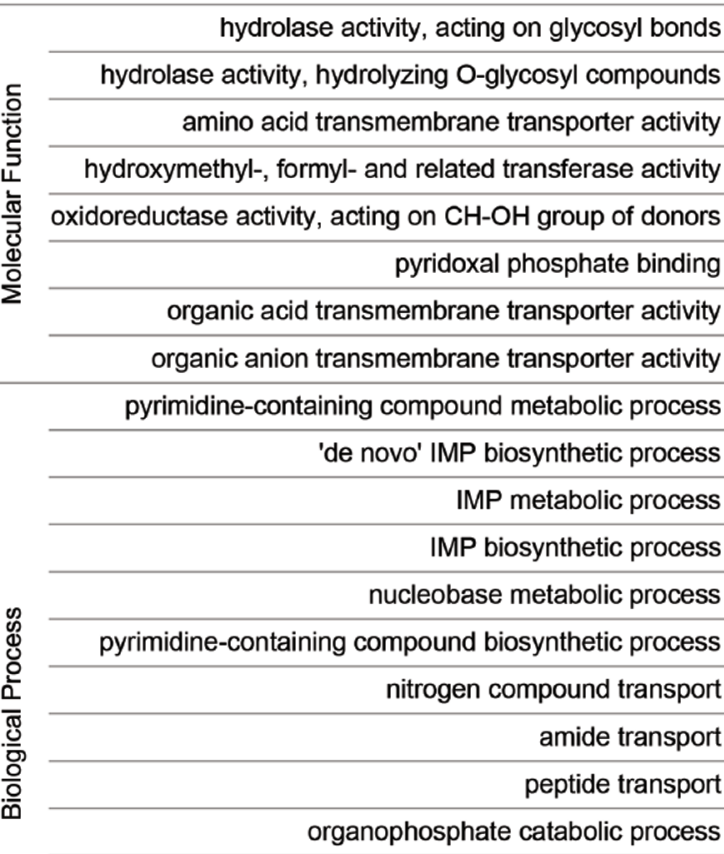

'de novo' pyrimidine nucleobase biosynthetic process pyrimidine nucleotide biosynthetic process amino acid transport pyrimidine nucleotide metabolic process

Amino acid permease/ SLC12A domain

Glycoside hydrolase, catalytic domain Oligopeptide/dipeptide ABC transporter, C-termina 6-phosphogluconate dehydrogenase, domain 2

Aminotransferase, class I/classI

Glycoside hydrolase superfamily

6-phosphogluconate dehydrogenase, NADP-binding

Biotin/lipoyl attachment Single hybrid moti Nitroreductase-like

Dihydrofolate reductase-like domain
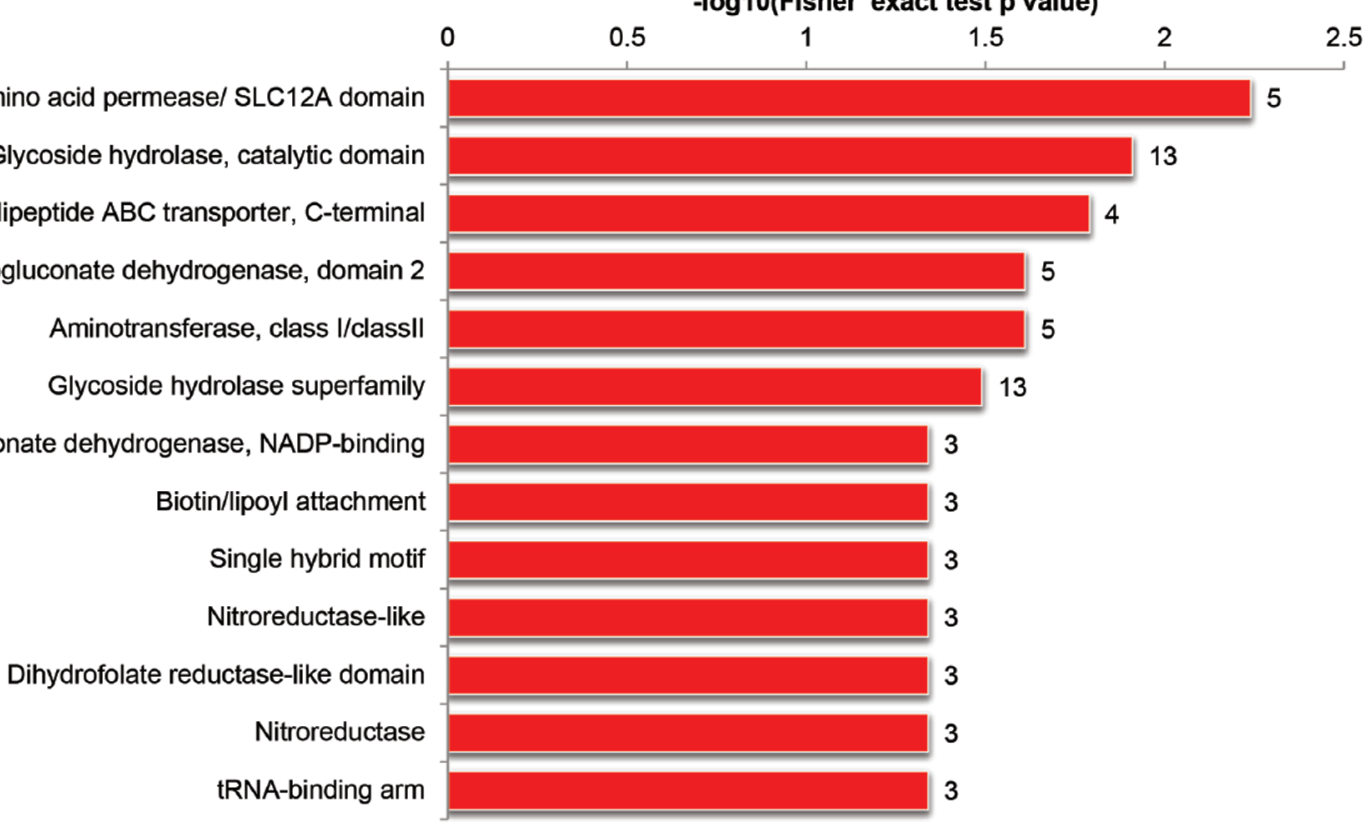

Figure 3. Histogram displaying Gene Ontology enrichment of differentially expressed proteins of Lactococcus lactis treated with glucose stress according to Fisher's exact test $P$-value. (A) Molecular functions and biological process enrichment of differentially expressed proteins in 60 versus $30 \mathrm{~m} M$ glucose treatment; (B) protein domain enrichment of differentially expressed proteins 60 versus $30 \mathrm{~m} M$ glucose treatment; (C) Kyoto Encyclopedia of Genes and Genomes (KEGG) enrichment of up-regulated proteins 60 versus $30 \mathrm{~m} M$ glucose treatment; and (D) KEGG enrichment of down-regulated proteins 60 versus $30 \mathrm{~m} M$ glucose treatment, respectively. Each related protein number is shown to the right of the bars. TCA = tricarboxylic acid; tRNA = transfer RNA; IMP = inosine monophosphate 
C

Ila00500 Starch and sucrose metabolism - Lactococcus lactis
subsp. lactis II1403
II1403
Ila00620 Pyruvate metabolism - Lactococcus lactis subsp. lactis
Ila00020 Citrate cycle (TCA cycle) - Lactococcus lactis subsp.
lactis II1403
Ila00280 Valine, leucine and isoleucine degradation -
Lactococcus lactis subsp. lactis II1403
Ila00010 Glycolysis / Gluconeogenesis - Lactococcus lactis
subsp. lactis II1403
Ila00360 Phenylalanine metabolism - Lactococcus lactis subsp.
lactis II03
Ila00380 Tryptophan metabolism - Lactococcus lactis subsp.
lactis II1403

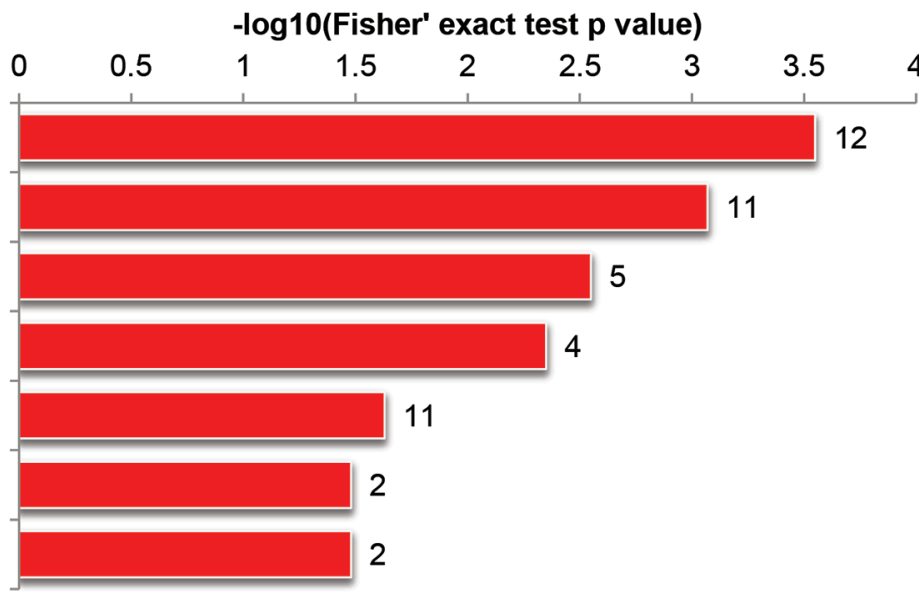

D

Ila00230 Purine metabolism - Lactococcus lactis subsp. lactis II1403

Ila00250 Alanine, aspartate and glutamate metabolism Lactococcus lactis subsp. lactis II1403

Ila02010 ABC transporters - Lactococcus lactis subsp. lactis II1403

Ila01501 beta-Lactam resistance - Lactococcus lactis subsp. lactis II1403

Ila00340 Histidine metabolism - Lactococcus lactis subsp. lactis II1403

Ila02024 Quorum sensing - Lactococcus lactis subsp. lactis II1403

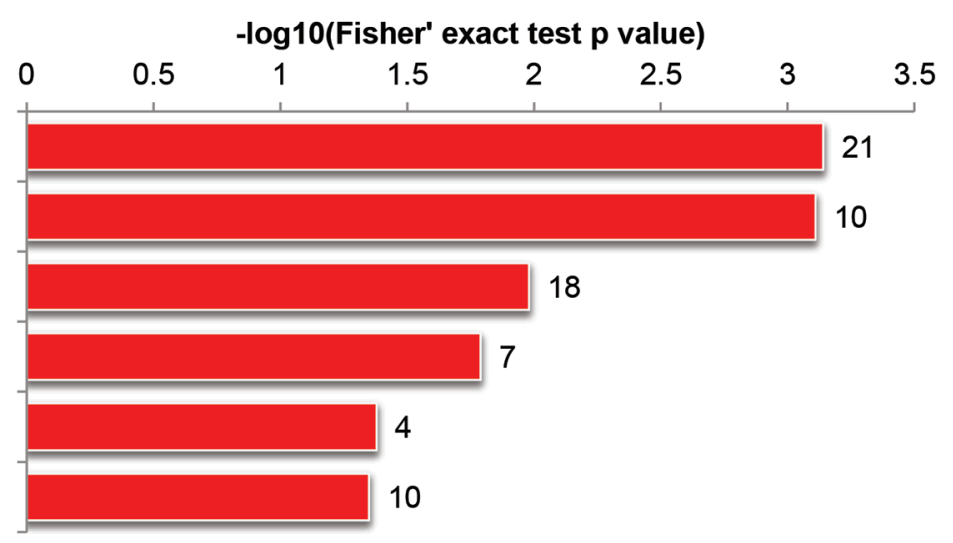

Figure 3 (Continued). Histogram displaying Gene Ontology enrichment of differentially expressed proteins of Lactococcus lactis treated with glucose stress according to Fisher's exact test $P$-value. (A) Molecular functions and biological process enrichment of differentially expressed proteins in 60 versus $30 \mathrm{~m} M$ glucose treatment; (B) protein domain enrichment of differentially expressed proteins 60 versus $30 \mathrm{~m} M$ glucose treatment; (C) Kyoto Encyclopedia of Genes and Genomes (KEGG) enrichment of up-regulated proteins 60 versus $30 \mathrm{~m} M$ glucose treatment; and (D) KEGG enrichment of down-regulated proteins 60 versus $30 \mathrm{~m} M$ glucose treatment, respectively. Each related protein number is shown to the right of the bars. TCA $=$ tricarboxylic acid; tRNA $=$ transfer RNA; IMP $=$ inosine monophosphate.

metabolism and nitrogen metabolism (valine, leucine and isoleucine degradation, phenylalanine metabolism, tryptophan metabolism, alanine, aspartate and glutamate metabolism, histidine metabolism, aminoacyltRNA biosynthesis) were enriched with proteins with both increased and decreased expression (Figure 3D). In addition, 9 aminoacyl-tRNA ligases (thrS, pheT, pheS, tyrS, glyQ, lysS, ileS, valS, and serS) were found to have decreased expression in this study. Our data confirmed the key role of metabolic alterations in the response to glucose stress in L. lactis.

\section{Functional Enrichment Cluster Analysis of Differentially Expressed Proteins}

The functional enrichment cluster analysis of differentially expressed proteins of L. lactis treated with glucose stress is demonstrated in Figure 4. Unsupervised hierarchical clustering of differentially expressed proteins in $60 \mathrm{~m} M$ versus $30 \mathrm{~m} M$ glucose treatment in the 4 groups included: quantitative category 1 (0 $<$ ratio $\leq 1 / 1.3$ and $P<0.05$ ), quantitative category $2(1 / 1.3<$ ratio $\leq 1 / 1.2$ and $P<0.05)$, quantitative category $3(1.2<$ ratio $\leq 1.3$ and $P<0.05)$, and quantitative category 4 (ratio $>1.3$ and $P<0.05$ ). The results indicated that down-regulated proteins (Q1, ratio $\leq 1 / 1.3)$ were most strongly associated with histidine metabolism, nitrogen metabolism, biosynthesis of amino acids, cysteine and methionine metabolism, alanine, aspartate and glutamate metabolism, quorum sensing, beta-lactam resistance, sulfur metabolism, purine metabolism, $\mathrm{ABC}$ transporters, and one carbon pool by folate. Up-regulated proteins (quantitative category 4 , ratio $>1.3$ ) were most strongly associated 
with pyruvate metabolism, the citrate cycle, starch and sucrose metabolism, glycolysis/gluconeogenesis, and propanoate metabolism.

\section{Metabonomic Analysis of L. lactis Treated with Different Concentrations of Glucose}

Metabonomic analysis of $L$. lactis after treatment with $5,10,15,30,60$, and $120 \mathrm{~m} M$ glucose, followed by metabolomic profiling, is illustrated in Figure 5. A total of 98 metabolites were detected, which were categorized as $28.57 \%$ acids (28), $23.46 \%$ amino acids (23), $17.35 \%$ sugars (17), $15.31 \%$ derivatives of ammonia or amine (15), $12.24 \%$ heterocycles of ketone and phenol (12), and $3.06 \%$ alcohols (3) (Supplemental Table S2 and Figure S1A; https://doi.org/10.3168/jds.2019-17810).

The investigation of metabolites was subjected to PCA. The PCA score plots show that each group was clearly discriminated from the others (Supplemental Figure S1B). The biplot of metabolites analyzed for the 5 principal components is shown in Supplemental
Figure S1C and Table S3 (https://doi.org/10.3168/jds .2019-17810). Principal components 1 and 2 accounted for $73.34 \%$ of the accumulative variance. As summarized in Supplemental Table S3, principal components 1 and 2 possessed 55 and 24 metabolites, respectively.

The PCA loading plots (Supplemental Figure S1C; https://doi.org/10.3168/jds.2019-17810) reveal that acetamide (metabolite 4), lactate (metabolite 10), proline (metabolite 17), glycerol (metabolite 23), pentanedioic acid (metabolite 43), ornithine (metabolite 50), lysine (metabolite 55), phosphoric acid (metabolite 56), fructofuranose (metabolite 58), glucose (metabolite 59), fructopyranose (metabolite 62), fructose (metabolite 67 ), sorbose (metabolite 68), mannopyranose (metabolite 70), galactose (metabolite 71), and ascorbic acid (metabolite 75) were clearly distinguishable from the other samples.

Acetamide is widely present in milk, beef, eggs, and roasted coffee beans, and is produced during food processing (Nault et al., 2020). Acetamide is formed from thermal breakdown of $\mathrm{N}$-acetylated metabolites (mono-
KEGG pathway

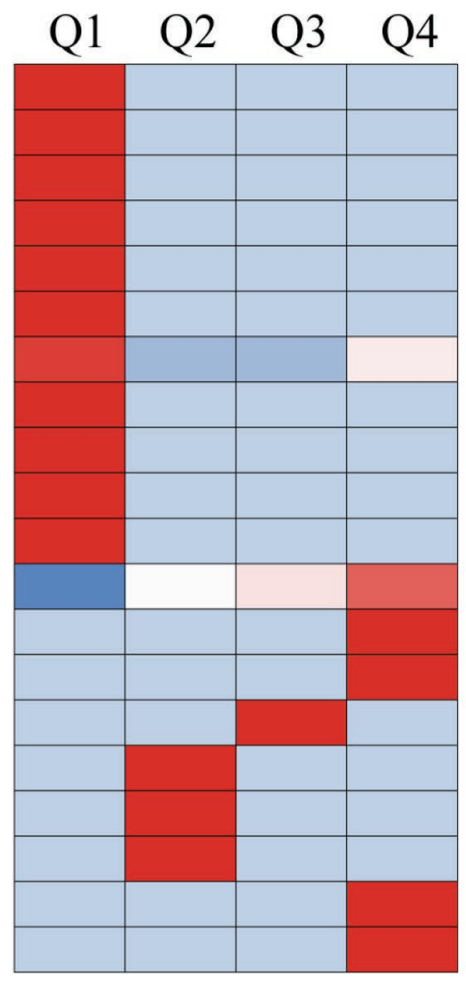

Zscore(- $\log 10$ (Fisher's exact test P value))

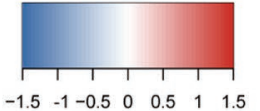

Histidine metabolism - Lactococcus lactis subsp. lactis II1403

Quorum sensing - Lactococcus lactis subsp. lactis ||1403

beta-Lactam resistance - Lactococcus lactis subsp. lactis II1403

Nitrogen metabolism - Lactococcus lactis subsp. lactis II1403

Sulfur metabolism - Lactococcus lactis subsp. lactis II1403

Cysteine and methionine metabolism - Lactococcus lactis subsp. lactis II1403

One carbon pool by folate - Lactococcus lactis subsp. lactis II1403

Biosynthesis of amino acids - Lactococcus lactis subsp. lactis II1403

ABC transporters - Lactococcus lactis subsp. lactis II1403

Purine metabolism - Lactococcus lactis subsp. lactis II1403

Alanine, aspartate and glutamate metabolism - Lactococcus lactis subsp. lactis II1403

Starch and sucrose metabolism - Lactococcus lactis subsp. lactis II1403

Glycolysis / Gluconeogenesis - Lactococcus lactis subsp. lactis II1403

Propanoate metabolism - Lactococcus lactis subsp. lactis II1403

Phenylalanine metabolism - Lactococcus lactis subsp. lactis II1403

Aminoacyl-tRNA biosynthesis - Lactococcus lactis subsp. lactis II1403

RNA polymerase - Lactococcus lactis subsp. lactis II1403

D-Alanine metabolism - Lactococcus lactis subsp. lactis II1403

Pyruvate metabolism - Lactococcus lactis subsp. lactis II1403

Citrate cycle (TCA cycle) - Lactococcus lactis subsp. lactis II1403

Figure 4. Kyoto Encyclopedia of Genes and Genomes (KEGG) pathway enrichment of differentially expressed proteins of Lactococcus lactis treated with glucose stress. Unsupervised hierarchical clustering of differentially expressed proteins 60 versus $30 \mathrm{~m} M$ glucose treatment in 4 groups included: quantitative category 1 (Q1; $0<$ ratio $\leq 1 / 1.3$ and $P<0.05)$, quantitative category $2(\mathrm{Q} 2 ; 1 / 1.3<$ ratio $\leq 1 / 1.2$ and $P<0.05)$, quantitative category $3(\mathrm{Q} 3 ; 1.2<$ ratio $\leq 1.3$ and $P<0.05)$, and quantitative category 4 (Q4; ratio $>1.3$ and $P<0.05)$. tRNA $=$ transfer RNA. 
saccharides $\quad N$-acetylglucosamine, $N$-acetylcysteine) and glycans during vaporization of GC-MS analysis (Vismeh et al., 2018). Therefore, acetamide can be involved in carbohydrate metabolism and considered to have harmful components. However, the acute toxicity of acetamide is very low and its lethal dose $50 \%$ value is in the grams per kilogram range (Kennedy, 2014). Amino acids such as L-proline, ornithine, and L-lysine are involved in amino acid metabolism and widely used in food additives and the feed industry (Jensen et al., 2015). Proline is generated from glutamate, through catalysis by glutamate 5-kinase, glutamate-5-semialdehyde dehydrogenase, delta-1-pyrroline-5-carboxylate synthetase, and pyrroline-5-carboxylate reductase. The glutamate 5-kinase was down-regulated 0.64 -fold at 60 $\mathrm{m} M$ versus $30 \mathrm{~m} M$. Ornithine is generated from glutamate, via catalysis by amino acid $N$-acetyltransferase, acetylglutamate kinase, acetylornithine deacetylase, and aminoacylase. Lysine is generated from aspartate, via catalysis by aspartate kinase, aspartate-semialdehyde dehydrogenase, succinyl-diaminopimelate desuccinylase, and diaminopimelate decarboxylase. Pentanedioic acid is a natural product in the catabolism of L-lysine via the aminovalerate pathway in bacteria ( $\mathrm{Yu}$ et al., 2017). Fructose, fructofuranose, fructopyranose, galactose, mannopyranose, sorbose, and glycerol are

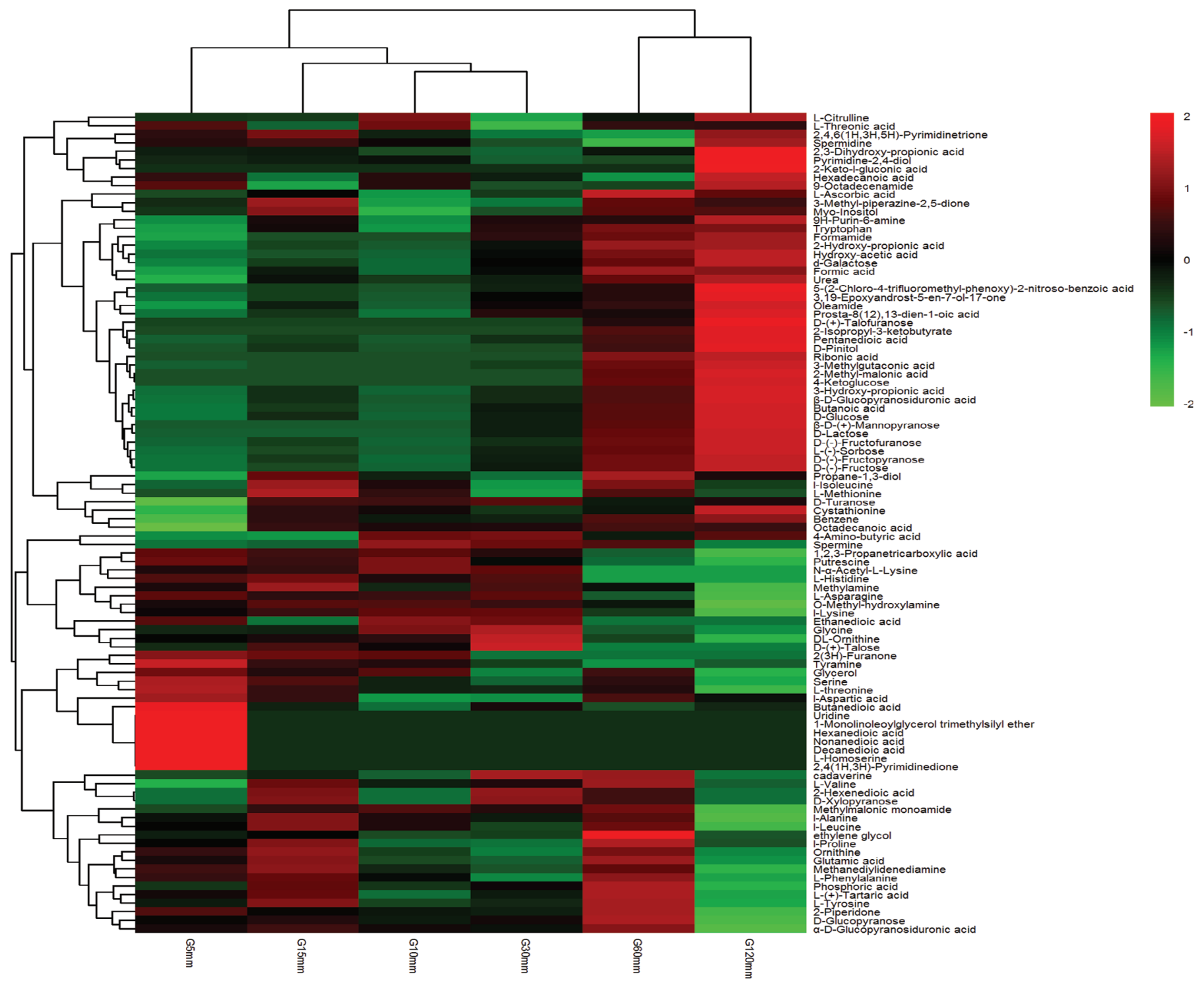

Figure 5. Heat map of unsupervised hierarchical clustering of all metabolites of Lactococcus lactis treated with glucose stress. Green and red indicate decreased and increased expression of metabolites, respectively. Values are scaled to the mean and SD of the metabolite level row (see color scale). 
involved in fructose, mannose, and galactose metabolism. Proline, glycerol, and sorbose can be classified as a compatible solute or osmoprotectant that plays important roles in response to various stresses (Liang et al., 2013). Fructose, sorbose, mannose, and galactose are monosaccharides that can be converted into or from glucose for catabolism (Sharma et al., 2014). Fructofuranose and fructopyranose are generated from fructose or sucrose (Gottschalk, 1945; Shiomi and Onodera, 1988). In water solution, beta-D-fructopyranose is the only crystalline form of D-fructose; in addition, there exists an interconversion between pyranose and furanose, and glucopyranose could be isomerized to fructofuranose (De et al., 2011). According to molecular mechanics calculations, the fructofuranose ring is a high-energy form (French, 1989). Many bacteria form primary carbohydrate reserves based on fructan (Meng and Fütterer, 2003). Ascorbic acid (vitamin C) is involved in ascorbate metabolism and is considered a promising anti-cancer agent (Shenoy et al., 2018). Ascorbic acid is produced from glucose-6P (plants) or glucose-1P (animals). Some studies showed that yeast cells can synthesize ascorbic acid via the pathway naturally used for D-erythroascorbic acid biosynthesis (Hancock et al., 2000), and bacteria can transform glucose to 2,5-keto-D-gluconic acid, which is used to produce the 2-keto-L-idonic acid, a precursor of L-ascorbic acid (Boudrant, 1990).

Metabolic pathways were enriched in differential metabolites, and the results showed an enrichment of 9 metabolic pathways (Figure 6). Up-regulated metabolites were enriched in fatty acid, fructose, mannose, galactose, propanoate, valine, leucine, and isoleucine metabolism. Moreover, down-regulated metabolites were enriched in nitrogen metabolism, which was in line with the proteomic results (Figure 3D).

\section{Effect of Glucose Stress on DNA Repair Process of $L$. lactis}

Mismatch repair pathway-related proteins, such DNA polymerase III (BN927_01049, 1.208-fold, BN927_02385, 1.275-fold), DNA-directed DNA polymerase (BN927_01802, 1.203), single-stranded DNAbinding protein (BN927_02507, 1.403, BN927_00106, 1.472), and priA were up-regulated. Ribosome-related proteins, DNA-directed RNA polymerase (rpoB, 0.813, rpoC, 0.805, rpoZ, 0.785), and ribonucleotide reductase (BN927_01203, 0.629) were down-regulated in L. lactis under high-glucose stress. Previous studies have shown that single-stranded DNA-binding protein genes were highly expressed under acid or erythromycin stress (Chitrakar et al., 2017; Li et al., 2018; Wu et al., 2018). Therefore, we inferred that RNA- and DNA-related regulatory mechanisms may also participate in the response to stress.

\section{Effects of Glucose Stress on Energy and Carbohydrate Metabolism of L. lactis}

Proteins with up-regulated expression were involved in energy metabolism such as starch and sucrose metabolism (12 proteins involved), pyruvate metabolism (11), glycolysis/gluconeogenesis (11), amino sugar and nucleotide sugar metabolism (8), citrate cycle (5), pentose phosphate pathway (5), and galactose metabolism (4). The increased expression of these energy generationrelated metabolism suggested that metabolic activity was enhanced in response to high-glucose condition.

The most efficient sugar uptake system in bacteria is the phosphotransferase (PTS) system, which includes histidine-containing phosphocarrier protein (HPr) plus enzyme I and enzyme II (Aleksandrzak-Piekarczyk et al., 2011). Enzyme II have 3 domains, IIA, IIB and IIC, and IIA and IIB, involved in phosphoryl group transfer, whereas domain IIC could form the translocation channel. The higher expression of proteins involved in PTS are beta-glucoside-specific (BN927_02039, 3.562-fold), 6-phospho-beta-glucosidase (BN927_02404, 1.294), cellobiose-specific IIB component (BN927_01839, 1.538), cellobiose-specific IIA component (BN927_01838, 1.715), lactose-specific IIA (BN927_02727, 1.204) maltose phosphorylase/trehalose phosphorylase (BN927_02410, 1.217), sucrose-specific PTS enzyme IIABC (BN927_01824, 1.228), mannose-specific IID (BN927_02452, 0.774), and trehalose-specific IIB (BN927_01823, 0.813). Moreover, beta-glucosidase (BN927_02040, 2.843-fold, BN927_02010, 1.265-fold) could hydrolyze glucosidic linkages in aryl-, amino-, or alkyl- $\beta$-D-glucosides, cyanogenic glucosides, and oligo- or disaccharides (Yeoman et al., 2010). Maltose produced from glycogen by catalysis of alphaamylase (BN927_02411, 1.3 fold), cytoplasmic alphaamylase (BN927_00812, 1.431), and neopullulanase (BN927_02673, 1.526) is an extracellular alpha-amylase (Jung et al., 2019). Some sugar utilization systems consist of operon structures without PTS. These operons contain genes involved in both sugar transport and metabolism (An et al., 2012). The beta-glucoside bgl operon played an essential role in the $\beta$-glucoside utilization in L. lactis (Bardowski et al., 1994) and was up-regulated 3.015-fold. The levels of glucose, lactose, fructose, and glucose in L. lactis increased in response to glucose. Díez et al. (2017) reported activation of glucose uptake by a cellobiose-specific PTS system in L. lactis in response to acid, ethanol, heat, and oxidative stress. Our data indicated that cellobioses, lactose, mannose, and sucrose also played important roles in 
$30 \mathrm{~mm} \quad 60 \mathrm{~mm}$

Fatty acid metabolism

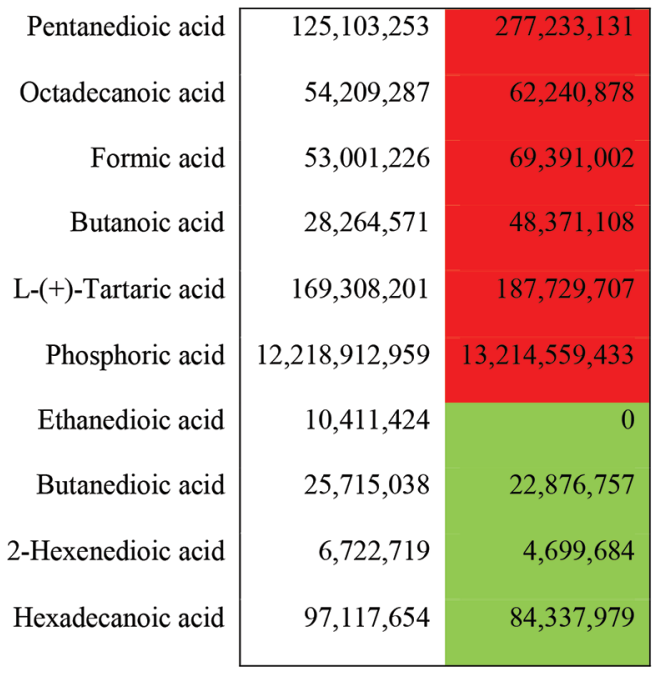

Ascorbate metabolism

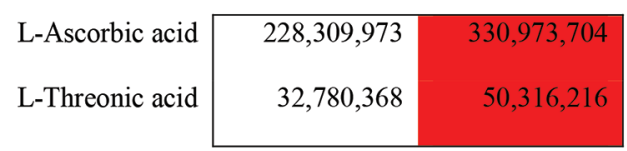

Fructose, mannose metabolism, and galactose metabolism

\begin{tabular}{r|rr|}
\cline { 2 - 3 } B-D-(+)-Mannopyranose & $934,861,035$ & $2,772,321,515$ \\
D-Galactose & 2310606659 & $3,985,551,099$ \\
D-Lactose & $25,277,257$ & $74,317,478$ \\
D-(-)-Fructose & $741,426,236$ & $1,643,439,914$ \\
L-(-)-Sorbose & $462,491,922$ & $1,188,526,255$ \\
Myo-Inositol & $77,167,778$ & $84,172,615$ \\
Glycerol & $1,843,971,561$ & $2,210,408,266$ \\
\hline
\end{tabular}

Propanoate metabolism

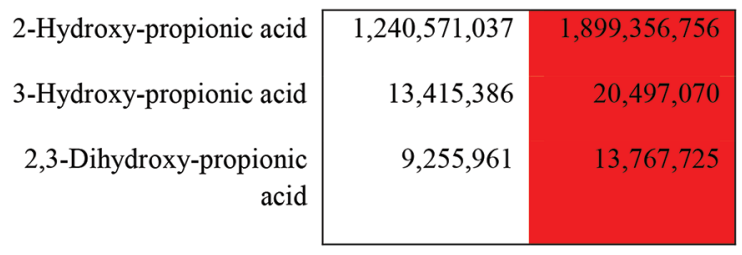

Increase

Decrease
Amino acids metabolism

$30 \mathrm{~mm}$

$60 \mathrm{~mm}$

Peak area

Alanine, aspartate, and glutamate metabolism

\begin{tabular}{c|r|r|}
\cline { 2 - 3 } L-Asparagine & $46,568,419$ & $25,351,354$ \\
L-Methionine & $72,376,585$ & $86,038,558$ \\
L-Alanine & $289,278,339$ & $318,999,616$ \\
\cline { 2 - 3 } & &
\end{tabular}

Valine, leucine, and isoleucine metabolism

\begin{tabular}{c|ccc}
\cline { 2 - 3 } L-Valine & $192,613,303$ & $208,349,611$ \\
L-Leucine & $286,946,521$ & $325,173,519$ \\
L-Isoleucine & $182,658,823$ & $205,706,434$ \\
\cline { 2 - 3 }
\end{tabular}

Arginine and proline metabolism

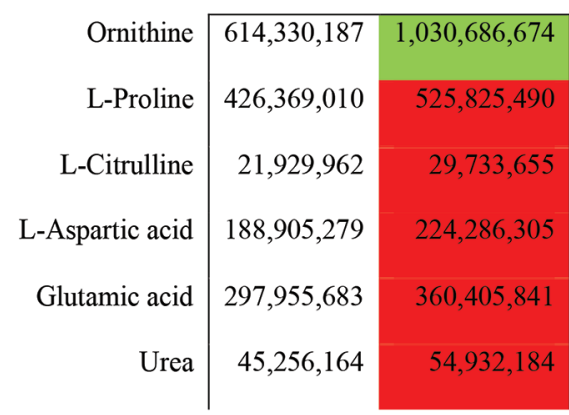

Lycine, serine, and threonine metabolism

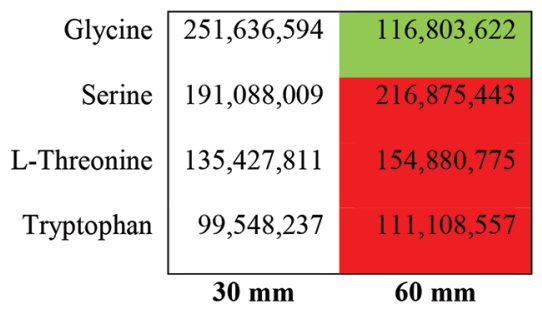

Biogenic amine metabolism Concentration $(\mathrm{mg} / \mathrm{g})$

\begin{tabular}{cr|r|} 
Putrescine & 0.5603 & 0.3515 \\
Cadaverine & 45.2326 & 43.7255 \\
Tyramine & 6.455 & 5.6657 \\
& \\
Spermine & 8.8203 & 8.1755 \\
Spermidine & 0.1437 & 0 \\
\cline { 2 - 3 }
\end{tabular}

Figure 6. Kyoto Encyclopedia of Genes and Genomes enrichment of the metabolites of Lactococcus lactis after treatment under a glucose stress. Green and red highlighting represent those metabolites with up-regulated and down-regulated expression, respectively. 
response to high-glucose and glucose-limited stress ( $\mathrm{He}$ et al., 2018; Yu et al., 2018). Lactose transport might be inhibited by high oxygen and acid stress (Larsen et al., 2016), which suggested that lactose transport is different in $L$. lactis in response to various stresses. Sucrose and maltose can protect membrane structures against fusion and leakage (Termont et al., 2006; Roy et al., 2016). Moreover, Zheng et al. (2019) demonstrated that maltose functions as a sugar protectant in Candida oleophila against heat stress. Therefore, the results indicated that the disaccharide maltose, derived from glucose for glycan biosynthesis, might serve as a potential osmoprotectant and potential carbon storage source in L. lactis.

Pyruvate metabolism was enhanced under glucose stress, and the expression of phosphoglycerate kinase (pgk, 1.261-fold), lactate dehydrogenase (BN927_01433, 1.317), aldehyde-alcohol dehydrogenase (BN927_02274, 1.435), acetate kinase (ackA, 1.411), and acylphosphatase (BN927_02165, 1.398) was markedly up-regulated. The levels of lactate, ethanol, and acetate increased in L. lactis in response to glucose, which indicated metabolic flux from pyruvate to lactate and acetate, leading to a decrease in the $\mathrm{pH}$ of the culture medium. Moreover, abundant acetyl-CoA was generated from pyruvate-flavodoxin oxidoreductase (BN927_01829, 1.465), dihydrolipoamide acetyltransferase (BN927_00448, 1.633), acetoin dehydrogenase (BN927_00449, 1.457, BN927_00450, 1.453), dihydrolipoyl dehydrogenase (BN927_00447, 1.54), phenylalanine [6-aminohexanoate-cyclic-dimer hydrolase (BN927_01315, 1.266)], tryptophan [3-ketoacyl-CoA thiolase @ acetyl-CoA acetyltransferase (BN927_01919, 1.786)], 6-aminohexanoate-cyclic-dimer hydrolase (BN927_01315, 1.266), histidinol-phosphate aminotransferase (hisC, 1.213), lysine, valine, leucine, isoleucine [dihydrolipoyl dehydrogenase (BN927_00447, 1.54)], 3-ketoacyl-CoA thiolase @ acetyl-CoA acetyltransferase (BN927_01919, 1.786), 2-hydroxy-3-oxopropionate reductase (BN927_00152, 1.27), branched-chain amino acid aminotransferase (BN927_00772, 1.224), and acetyl-CoA flux to the citrate cycle [dihydrolipoyl dehydrogenase (BN927_00447, 1.54)]. 3-Ketoacyl-CoA thiolase @ acetyl-CoA acetyltransferase catalyzes the final step of fatty acid oxidation to release acetyl-CoA. Moreover, the levels of acetyl-CoA precursor substances such as phenylalanine, tryptophan, lysine, valine, leucine, and isoleucine are increased in L. lactis in response to glucose.

\section{Effect of Glucose Stress on AA Metabolism of L. lactis}

Proteins with altered expression in the amino acid metabolic pathway included aspartate (aspartate ami- notransferase (BN927_01322, 1.205-fold), adenylosuccinate synthetase (purA, 0.628), adenylosuccinate lyase (BN927_02371, 0.552), glutamate [glutamate synthase (BN927_00775, 0.391, BN927_00774, 0.403)], histidine [imidazole glycerol phosphate synthase (hisF, 0.58)], histidinol dehydrogenase (hisD, 0.424), imidazole glycerol phosphate dehydratase (hisB, 0.413), ATP phosphoribosyltransferase (hisZ, 0.406), histidinol-phosphate aminotransferase (hisC, 1.213), and arginine [carbamoyl-phosphate synthase (carA, 0.55, carB, 0.512)]. As previously shown, histidinol phosphatase (hisK) was up-regulated in L. lactis ssp. lactis in response to gradient freezing stress (Lu et al., 2019). Lysine biosynthesis might be responsible for the high-glucose stress tolerance of C. glycerinogenes (Yang et al., 2018). Arginine could protect yeast and L. lactis cells against ethanol stress by maintaining the integrity of the cell wall and cytoplasmic membranes (Díez et al., 2017).

The level of glutamate increased under glucose stress, which was predicted to be the main factor that acts as a switch for the regulation of cell growth and carbon accumulation. Considering the high levels of glutamate in the cells under glucose stress (Shin et al., 2015), the down-regulated expression of glutamate synthase (BN927_00775, BN927_00774) and glutamine synthetase (BN927_00137) suggested that the cells readjust nitrogen metabolism by the GS/GOGAT pathway (Ma et al., 2009). Moreover, glutamine synthetase might be part of a general adaptation strategy of lactic acid bacteria to the dairy environment (van Hylckama Vlieg et al., 2006).

Down-regulation of the expression level of protein involved in methionine metabolism, such as methionine biosynthesis and transport regulator MtaR (BN927_01938, 0.704-fold), methionine synthase (BN927_02656, 0.49), and methionine, import ATPbinding protein MetN (metN, 0.472). Previous work showed that the enzymes involved in methionine metabolism increased in response to elevated temperatures in L. lactis, which could directly improve the production of the key cheese flavor compound methanethiol (van Hylckama Vlieg et al., 2006).

It was observed that the expression of serine protease DegP/Htr (BN927_00315, 1.929-fold) was significantly up-regulated. The HtrA is a housekeeping surface protease in L. lactis and is a critical factor for bacterial survival under environmental stress conditions (Poquet et al., 2000; Foucaud-Scheunemann and Poquet, 2003). The HtrA proteins are known bifunctional proteins that function as both molecular chaperones and proteases (conserved from bacteria to humans). Their proteolytic activity (in most cases chaperone activity) efficiently counteracted the consequences of stressful conditions (Kim et al., 2008; Singh et al., 2011; Zarzecka et al., 
2018). The HtrA was up-regulated in response to acid stress tolerance in L. lactis (Wu et al., 2018).

\section{Effects of Glucose Stress on tRNA Synthetases and Biosynthesis of Biogenic Amine of L. lactis}

The levels of many aminoacyl-tRNA biosynthesisrelated metabolites such as threonine, phenylalanine, tyrosine, isoleucine, valine, serine, which are the precursors of amino acid-tRNA, were increased. In contrast, aminoacyl-tRNA ligases (thrS, 0.8-fold; pheT, 0.765; pheS, 0.709; tyrS, 0.824; glyQ, 0.818; lysS, 0.795; ileS, 0.789; valS, 0.772; and serS, 0.8) were largely decreased in response to high glucose. The results showed that the activity of aminoacyl-tRNA synthetase was disturbed by glucose stress and that protein biosynthetic processes were suppressed.

Biogenic amines, including cadaverine, tyramine, putrescine, spermine, and spermidine, are derived from the microbial decarboxylation of the corresponding amino acids through substrate-specific decarboxylase enzymes (Loukou and Zotou, 2003). Lysine decarboxylation results in the formation of cadaverine. Tyrosine decarboxylation occurs to generate tyramine. Transformation of glutamic acid to arginine is found, followed by the formation of putrescine, spermine, and spermidine. The levels of lysine, putrescine, cadaverine, tyramine, spermine, and spermidine are decreased in $L$. lactis in response to glucose. Decarboxylation of amino acids is the main way in which enhanced lactic acid bacteria survival arises under environmental stress; moreover, the antiporter/decarboxylase system is an indirect proton pump analogous to other mechanisms providing energy and protecting from acidic conditions (Papadimitriou et al., 2016). Biogenic amines are considered potentially hazardous substances in fermented foods (Park et al., 2019). Tyramine can react with nitrite to generate C-nitrosation, which is mutagenic in the human stomach (González-Jiménez et al., 2017). Putrescine is associated with neurologic disorders and neurotoxicity (Fernandes et al., 2018), and a higher level of putrescine is produced by L. lactis (del Rio et al., 2016). Spermine and spermidine are cytotoxic and cause intestinal cell necrosis (del Rio et al., 2018).

\section{Analysis of the Metabolic Network of L. lactis}

To further explore the comprehensive influence of proteins and metabolites on the response to glucose treatment, the metabolic network, which consisted of a combination of proteomic and metabonomic data, was established as shown in Figure 7.
Up-regulated metabolic pathways included starch and sucrose metabolic transformation of monosaccharide into polysaccharides, especially enhanced maltose metabolism, glycolysis conversion of glucose into pyruvate and acetic acid, pyruvate oxidation to generate acetyl-CoA, and amino acid metabolic transformation of leucine, valine, and isoleucine into acetyl-CoA connected via fatty acid metabolism. Phenylalanine and tryptophan could also generate acetyl-CoA. The extracellular level of glucose could affect metabolic flux in the stress response of L. lactis (Duwat et al., 2000). High-glucose stress can enhance glycolysis metabolism in C. glycerinogenes (Yang et al., 2018). Research has shown that the metabolic flux is increased in glycolysis, the citrate cycle, and pyruvate metabolism in L. lactis in response to glucose stress. This result is consistent with a previous study showing that Lactobacillus plantarum activation consists of an increase in the metabolism of carbohydrates (via glycolysis, the citric acid cycle, and pyruvate metabolism) in response to glucose-limited stress (He et al., 2018).

Among the down-regulated metabolic pathways, metabolic flux was decreased for histidine, lysine, alanine, aspartate, glutamate, and arginine metabolism in L. lactis in response to glucose stress. This led to reduced biogenic amine production, which is in agreement with a previous study indicating that Lactobacillus plantarum activation increases amino acid catabolism (via serine, threonine, and histidine metabolism, lysine degradation, and arginine responses to glucose-limited stress; He et al., 2018; Pan et al., 2019). Saccharomyces cerevisiae metabolic pathway regulation of the citrate cycle and alanine, aspartate, and glutamate metabolisms occur in response to ethanol stress (Ming et al., 2019).

Lactic acid bacteria could encounter a series of environmental stresses in the manufacture of fermented dairy foods. Consequently, the organism has advantages in terms of high sugar-uptake rates, deamination and decarboxylation of amino acids, and higher acid tolerance allowing survival under harsh stress conditions (Zuljan et al., 2016; Sauer et al., 2017). During acid stress, additional energy is provided by the upregulation of carbohydrate metabolism, and enhanced regulation of amino acid metabolism and transport to maintain $\mathrm{pH}$ homeostasis and ATP generation (Mills et al., 2011). The levels of glycolytic enzymes (pyruvate oxidase and phosphate acetyltransferase) increase in response to acid, thermal, and osmotic stresses, leading to improved synthesis of glycogen, lysine, and diacetyl/acetoin (Zhu et al., 2019). Previous research suggested a link between the heat shock response and acid resistance in L. lactis (Sanders et al., 1999). Under 


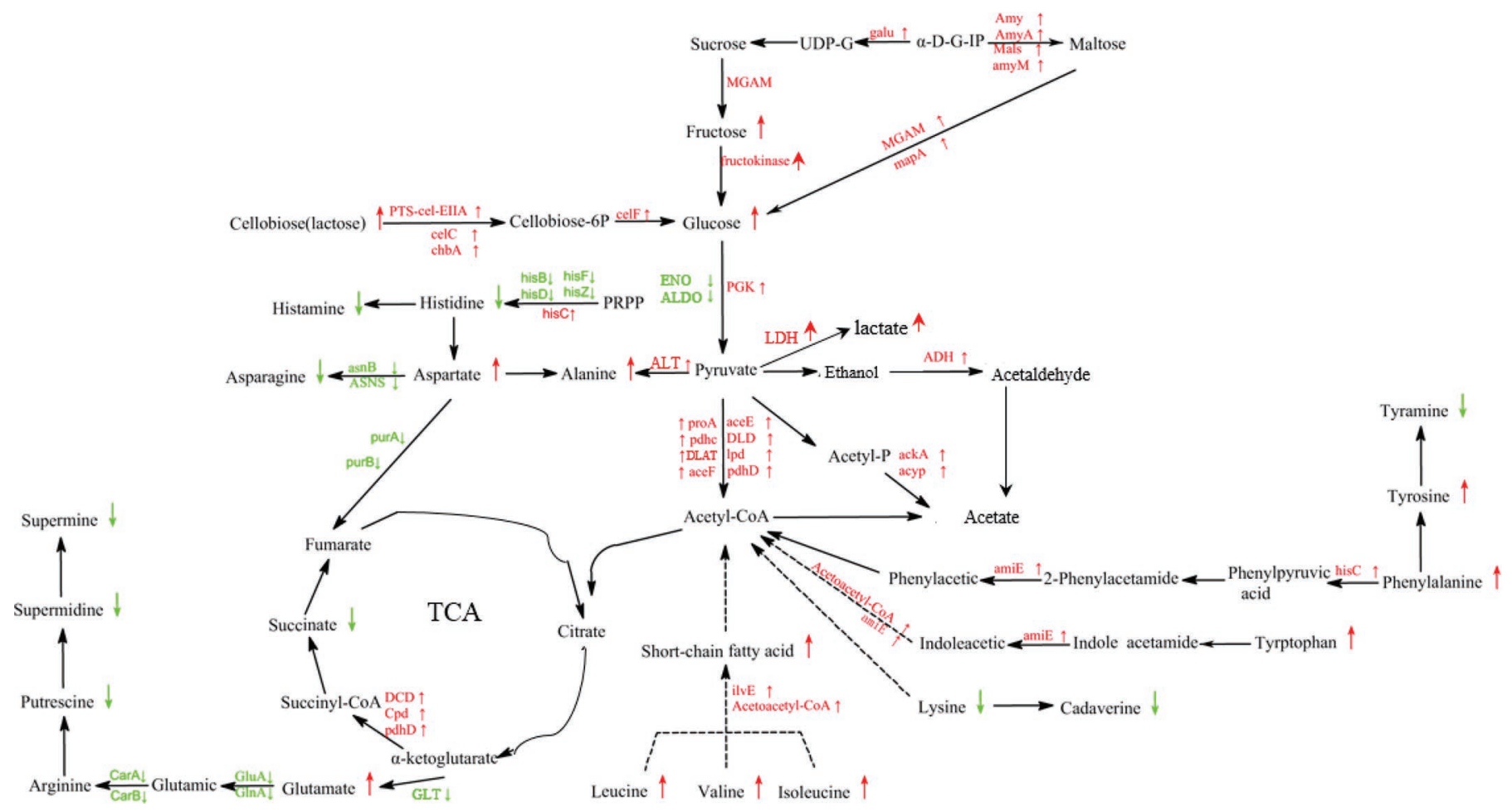

Figure 7. Metabolic profiles of Lactococcus lactis treated with glucose stress. Green and red arrows indicate those proteins and metabolites with up-regulated and down-regulated expression, respectively. UDP-G = uridine diphosphate glucose; $\alpha$-D-G-1P $=\alpha$-D-glucose-1-phosphate; Cellobiose-6P = cellobiose-6- phosphate; PRPP = 5-phosphoribosyl 1-pyrophosphate; TCA = tricarboxylic acid; galu = UTP-glucose-1phosphate uridylyltransferase (BN927_00701); Amy = alpha-amylase (BN927_02411); AmyA = alpha-amylase (BN927_00812); amyM = alphaamylase (BN927_02412); Mals = neopullulanase (BN927_02673); MGAM = maltose phosphorylase/trehalose phosphorylase (BN927_02410); mapA = amylomaltase (BN927_02678); PTS-cel-EIIA = PTS system, cellobiose-specific IIA (BN927_01838); celC = PTS system, lactose-specific IIA component (BN927_02727); chbA = PTS system, sucrose-specific IIA component (BN927_01824); celF = 6-phospho-beta-glucosidase (BN927_02404); hisB = imidazole glycerol-phosphate dehydratase; hisC = histidinol-phosphate aminotransferase; hisD = histidinol dehydrogenase; hisF = imidazole glycerol phosphate synthase; hisZ = ATP phosphoribosyltransferase; ENO = enolase (eno); ALDO = fructose-bisphosphate aldolase (BN927_02543); PGK = phosphoglycerate kinase (pgk); asnB = asparagine synthetase [glutamine-hydrolyzing] (BN927_01104); ASNS = asparagine synthetase [glutamine-hydrolyzing] (BN927_00164); ALT $=$ aspartate aminotransferase $($ BN927_01322); LDH $=$ lactate dehydrogenase (BN927_01808); $\mathrm{ADH}=$ alcohol dehydrogenase (BN927_01947); purA = adenylosuccinate synthetase; purB = adenylosuccinate lyase (BN927_02371); proA = pyruvate-flavodoxin oxidoreductase (BN927_01829); pdhc = acylphosphatase (BN927_02165); DLAT = dihydrolipoamide acetyltransferase component of pyruvate dehydrogenase (BN927_00448); aceF = acetoin dehydrogenase (BN927_00449); aceE = acetoin dehydrogenase (BN927_00450); DLD = phosphate acetyltransferase (BN927_02391); lpd = biotin carboxyl carrier protein of acetyl-CoA carboxylase (BN927_02597); pdhD = dihydrolipoyl dehydrogenase (BN927_00447); ackA = acetate kinase; acyp = acylphosphatase (BN927_02165); DCD = succinyl-CoA synthetase (BN927_01327); Cpd = muramoyltetrapeptide carboxypeptidase (BN927_02457); ilvE = branched-chain-amino-acid aminotransferase (BN927_00772); Acetoacetyl-CoA = acetyl-CoA acetyltransferase (BN927_01919); amiE = 6-aminohexanoate-cyclic-dimer hydrolase (BN927_01315); CarA = carbamoyl-phosphate synthase; CarB = carbamoyl-phosphate synthase; GluA = glutamate transport ATP-binding protein (BN927_01378); GlnA = glutamine synthetase (BN927_00137); GLT = glutamate synthase $(\mathrm{NADPH})$.

oxidative stress, the levels of alcohol dehydrogenase and pyruvate dehydrogenase complex are reduced, leading to decreased synthesis of lactic acid and ethanol in lactic acid bacteria. Lactococcus lactis possesses a citrate/ lactate antiporter, which could metabolize citrate and synthesize acetic acid and ATP under carbohydrate starvation conditions (Papadimitriou et al., 2016). Lactococcus lactis has an advantage in terms of a higher glycine betaine uptake function and membrane-associated proteins (FtsH and HtrA) imparting resistance to osmotic stress (van de Guchte et al., 2002; Serrazanetti et al., 2009).

\section{CONCLUSIONS}

The response mechanisms underlying the metabolic adaptation of L. lactis to higher levels of glucose were elucidated using a combined proteomic and metabonomic method. The results showed that the RNA and DNA mismatch repair process and energy metabolism were enhanced. Also, the metabolic flux from glucose to pyruvate, lactate, acetate, and maltose was improved. The nitrogen metabolism-associated metabolic pathway decreased, which might be related to the inhibition of the production of biogenic amines. This study 
constructed a model for global response mechanisms to glucose stress and identified the possibility that reduced formation of biogenic amines improves level of sugar in the dairy fermentation industry. Moreover, according to the demand for industrial production, sugar concentration in fermented foods should be higher than the set value, which is dependent on bacterial strain and biogenic amine yield.

\section{ACKNOWLEDGMENTS}

This work was supported by grants from National Natural Science Foundation of China (Beijing; 31501449, 2017YFD0400303, SKLFNS-KF-201912, TD13-5015, and 18JCTPJC56800). The authors have not stated any conflicts of interest.

\section{REFERENCES}

Aleksandrzak-Piekarczyk, T., J. Polak, B. Jezierska, P. Renault, and J. Bardowski. 2011. Genetic characterization of the CcpA-dependent, cellobiose-specific PTS system comprising CelB, PtcB and PtcA that transports lactose in Lactococcus lactis IL1403. Int. J. Food Microbiol. 145:186-194. https://doi.org/10.1016/j.ijfoodmicro 2010.12 .011$.

An, C. L., M. K. Kim, T. H. Kang, J. Kim, H. Kim, and H. D. Yun. 2012. Cloning and biochemical analysis of $\beta$-glucoside utilization (bgl) operon without phosphotransferase system in Pectobacterium carotovorum subsp. carotovorum LY34. Microbiol. Res. 167:461469. https://doi.org/10.1016/j.micres.2012.03.004.

Bardowski, J., S. D. Ehrlich, and A. Chopin. 1994. BglR protein, which belongs to the BglG family of transcriptional antiterminators, is involved in beta-glucoside utilization in Lactococcus lactis. J. Bacteriol. 176:5681-5685. https://doi.org/10.1128/JB.176.18 $.5681-5685.1994$.

Boudrant, J. 1990. Microbial processes for ascorbic acid biosynthesis: A review. Enzyme Microb. Technol. 12:322-329. https://doi.org/ 10.1016/0141-0229(90)90159-N.

Chitrakar, I., D. M. Kim-Holzapfel, W. Zhou, and J. B. French. 2017. Higher order structures in purine and pyrimidine metabolism. J. Struct. Biol. 197:354-364. https://doi.org/10.1016/j.jsb.2017.01 .003 .

Darby, T. M., J. A. Owens, B. J. Saeedi, L. Luo, J. D. Matthews, B. S. Robinson, C. R. Naudin, and R. M. Jones. 2019. Lactococcus lactis subsp. cremoris is an efficacious beneficial bacterium that limits tissue injury in the intestine. iScience 12:356-367.

De, S., S. Dutta, and B. Saha. 2011. Microwave assisted conversion of carbohydrates and biopolymers to 5-hydroxymethylfurfural with aluminium chloride catalyst in water. Green Chem. 13:2859-2868. https://doi.org/10.1039/c1gc15550d.

del Rio, B., V. Ladero, B. Redruello, D. M. Linares, M. Fernández, M. C. Martín, and M. A. Alvarez. 2015a. Lactose-mediated carbon catabolite repression of putrescine production in dairy Lactococcus lactis is strain dependent. Food Microbiol. 48:163-170. https://doi .org/10.1016/j.fm.2014.11.018.

del Rio, B., B. Redruello, V. Ladero, M. Fernandez, M. C. Martin, and M. A. Alvarez. 2016. Putrescine production by Lactococcus lactis subsp. cremoris CECT 8666 is reduced by $\mathrm{NaCl}$ via a decrease in bacterial growth and the repression of the genes involved in putrescine production. Int. J. Food Microbiol. 232:1-6. https://doi.org/ 10.1016/j.ijfoodmicro.2016.05.010.

del Rio, B., B. Redruello, D. M. Linares, V. Ladero, P. Ruas-Madiedo, M. Fernandez, M. C. Martin, and M. A. Alvarez. 2018. Spermine and spermidine are cytotoxic towards intestinal cell cultures, but are they a health hazard at concentrations found in foods? Food
Chem. 269:321-326. https://doi.org/10.1016/j.foodchem.2018.06 .148 .

del Rio, B., B. Redruello, M. C. Martin, M. Fernandez, A. de Jong, O. P. Kuipers, V. Ladero, and M. A. Alvarez. 2015b. Transcriptome profiling of Lactococcus lactis subsp. cremoris CECT 8666 in response to agmatine. Genom. Data 7:112-114. https://doi.org/10 $.1016 /$ j.gdata.2015.12.003

Díez, L., A. Solopova, R. Fernández-Pérez, M. González, C. Tenorio, O. P. Kuipers, and F. Ruiz-Larrea. 2017. Transcriptome analysis shows activation of the arginine deiminase pathway in Lactococcus lactis as a response to ethanol stress. Int. J. Food Microbiol. 257:41-48. https://doi.org/10.1016/j.ijfoodmicro.2017.05.017.

Duwat, P., B. Cesselin, S. Sourice, and A. Gruss. 2000. Lactococcus lactis, a bacterial model for stress responses and survival. Int. J. Food Microbiol. 55:83-86. https://doi.org/10.1016/S0168 -1605(00)00179-3.

Fernandes, J., J. D. Chandler, K. H. Liu, K. Uppal, Y.-M. Go, and D. P. Jones. 2018. Putrescine as indicator of manganese neurotoxicity: Dose-response study in human SH-SY5Y cells. Food Chem. Toxicol. 116:272-280. https://doi.org/10.1016/j.fct.2018.04.042.

Forslund, S. K., M. Kaduk, and E. L. L. Sonnhammer. 2019. Evolution of protein domain architectures. Pages 469-504 in Evolutionary Genomics: Statistical and Computational Methods. M. Anisimova, ed. Springer, New York, NY.

Foucaud-Scheunemann, C., and I. Poquet. 2003. HtrA is a key factor in the response to specific stress conditions in Lactococcus lactis. FEMS Microbiol. Lett. 224:53-59. https://doi.org/10.1016/S0378 -1097(03)00419-1.

French, A. D. 1989. Chemical and physical properties of fructans. J. Plant Physiol. 134:125-136. https://doi.org/10.1016/S0176 -1617(89)80044-6.

Gonzalez, E. E., D. Olson, and K. Aryana. 2017. Short communication: Salt tolerance of Lactococcus lactis R-604 as influenced by mild stresses from ethanol, heat, hydrogen peroxide, and UV light. J. Dairy Sci. 100:4290-4293. https://doi.org/10.3168/jds.2016 -12088 .

González-Jiménez, M., J. Arenas-Valgañón, M. P. García-Santos, E. Calle, and J. Casado. 2017. Mutagenic products are promoted in the nitrosation of tyramine. Food Chem. 216:60-65. https://doi .org/10.1016/j.foodchem.2016.08.006.

Gottschalk, A. 1945. Yeast hexokinase and its substrates d-fructofuranose and D-glucose. Nature 156:540-541. https://doi.org/10.1038/ $156540 \mathrm{a} 0$.

Hancock, R. D., J. R. Galpin, and R. Viola. 2000. Biosynthesis of L-ascorbic acid (vitamin C) by Saccharomyces cerevisiae. FEMS Microbiol. Lett. 186:245-250,

He, Q., C. Cao, W. Hui, J. Yu, H. Zhang, and W. Zhang. 2018. Genomic resequencing combined with quantitative proteomic analyses elucidate the survival mechanisms of Lactobacillus plantarum P-8 in a long-term glucose-limited experiment. J. Proteomics 176:37-45. https://doi.org/10.1016/j.jprot.2018.01.013.

Huang, H., Z. Luo, S. Qi, J. Huang, P. Xu, X. Wang, L. Gao, F. Li, J. Wang, W. Zhao, W. Gu, Z. Chen, L. Dai, J. Dai, and Y. Zhao. 2018. Landscape of the regulatory elements for lysine 2-hydroxyisobutyrylation pathway. Cell Res. 28:111-125. https://doi.org/10 $.1038 / \mathrm{cr} .2017 .149$.

Jensen, J. V. K., D. Eberhardt, and V. F. Wendisch. 2015. Modular pathway engineering of Corynebacterium glutamicum for production of the glutamate-derived compounds ornithine, proline, putrescine, citrulline, and arginine. J. Biotechnol. 214:85-94. https:/ /doi.org/10.1016/j.jbiotec.2015.09.017.

Jung, J.-H., Y.-K. An, S.-Y. Son, S.-Y. Jeong, D.-H. Seo, M.-K. Kim, and C.-S. Park. 2019. Characterization of a novel extracellular

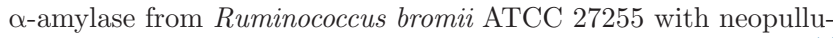
lanase-like activity. Int. J. Biol. Macromol. 130:605-614. https:// doi.org/10.1016/j.ijbiomac.2019.03.003.

Kennedy, G. L. 2014. Acetamide. Pages 24-25 in Encyclopedia of Toxicology (Third Edition). P. Wexler, ed. Academic Press, London, UK.

Kim, D. Y., E. Kwon, Y.-K. Shin, D.-H. Kweon, and K. K. Kim. 2008. The mechanism of temperature-induced bacterial HtrA activation. 
J. Mol. Biol. 377:410-420. https://doi.org/10.1016/j.jmb.2007.12 .078 .

Larsen, N., B. Brøsted Werner, and L. Jespersen. 2016. Transcriptional responses in Lactococcus lactis subsp. cremoris to the changes in oxygen and redox potential during milk acidification. Lett. Appl. Microbiol. 63:117-123. https://doi.org/10.1111/lam.12596.

Li, W., S. Zhang, X. Wang, J. Yu, Z. Li, W. Lin, and X. Lin. 2018. Systematically integrated metabonomic-proteomic studies of Escherichia coli under ciprofloxacin stress. J. Proteomics 179:61-70. https://doi.org/10.1016/j.jprot.2018.03.002.

Liang, X., L. Zhang, S. K. Natarajan, and D. F. Becker. 2013. Proline mechanisms of stress survival. Antioxid. Redox Signal. 19:9981011. https://doi.org/10.1089/ars.2012.5074.

Linares, D. M., B. del Río, V. Ladero, B. Redruello, M. C. Martín, M. Fernández, and M. A. Alvarez. 2013. The putrescine biosynthesis pathway in Lactococcus lactis is transcriptionally regulated by carbon catabolic repression, mediated by CcpA. Int. J. Food Microbiol. 165:43-50. https://doi.org/10.1016/j.ijfoodmicro.2013.04.021.

Loukou, Z., and A. Zotou. 2003. Determination of biogenic amines as dansyl derivatives in alcoholic beverages by high-performance liquid chromatography with fluorimetric detection and characterization of the dansylated amines by liquid chromatographyatmospheric pressure chemical ionization mass spectrometry. J. Chromatogr. A 996:103-113. https://doi.org/10.1016/S0021 -9673(03)00558-2.

Lu, J., L. Cui, S. Lin, L. Hao, N. Cao, J. Yi, X. Liu, L. Lu, and Q. Kang. 2019. Short communication: Global transcriptome analysis of Lactococcus lactis ssp. lactis in response to gradient freezing. J. Dairy Sci. 102:3933-3938. https://doi.org/10.3168/jds.2018-15972.

Ma, H., F. C. Boogerd, and I. Goryanin. 2009. Modelling nitrogen assimilation of Escherichia coli at low ammonium concentration. J. Biotechnol. 144:175-183. https://doi.org/10.1016/j.jbiotec.2009 .09 .003

Meng, G., and K. Fütterer. 2003. Structural framework of fructosyl transfer in Bacillus subtilis levansucrase. Nat. Struct. Biol. 10:935941. https://doi.org/10.1038/nsb974.

Mills, S., C. Stanton, G. F. Fitzgerald, and R. P. Ross. 2011. Enhancing the stress responses of probiotics for a lifestyle from gut to product and back again. Microb. Cell Fact. 10(Suppl 1):S19. https: //doi.org/10.1186/1475-2859-10-S1-S19.

Ming, M., X. Y. Wang, L. L. Lian, H. Zhang, W. X. Gao, B. Zhu, and D. W. Lou. 2019. Metabolic responses of Saccharomyces cerevisiae to ethanol stress using gas chromatography-mass spectrometry. Mol. Omics 15:216-221. https://doi.org/10.1039/C9MO00055K.

Nault, R., B. Bals, F. Teymouri, M. B. Black, M. E. Andersen, P. D. McMullen, S. Krishnan, N. Kuravadi, N. Paul, S. Kumar, K. Kannan, K. C. Jayachandra, L. Alagappan, B. D. Patel, K. T. Bogen, B. B. Gollapudi, J. E. Klaunig, T. R. Zacharewski, and V. Bringi. 2020. A toxicogenomic approach for the risk assessment of the food contaminant acetamide. Toxicol. Appl. Pharmacol. 388:114872. https://doi.org/10.1016/j.taap.2019.114872.

Pan, L., J. Yu, D. Ren, C. Yao, Y. Chen, and B. Menghe. 2019. Metabolomic analysis of significant changes in Lactobacillus casei Zhang during culturing to generation 4,000 under conditions of glucose restriction. J. Dairy Sci. 102:3851-3867. https://doi.org/10.3168/ jds.2018-15702.

Papadimitriou, K., A. Alegria, P. A. Bron, M. de Angelis, M. Gobbetti, M. Kleerebezem, J. A. Lemos, D. M. Linares, P. Ross, C. Stanton, F. Turroni, D. van Sinderen, P. Varmanen, M. Ventura, M. Zuniga, E. Tsakalidou, and J. Kok. 2016. Stress physiology of lactic acid bacteria. Microbiol. Mol. Biol. Rev. 80:837-890. https:/ /doi.org/10.1128/MMBR.00076-15.

Park, Y. K., J. H. Lee, and J.-H. Mah. 2019. Occurrence and reduction of biogenic amines in traditional Asian fermented soybean foods: A review. Food Chem. 278:1-9. https://doi.org/10.1016/j.foodchem 2018.11.045.

Poquet, I., V. Saint, E. Seznec, N. Simoes, A. Bolotin, and A. Gruss. 2000. HtrA is the unique surface housekeeping protease in Lactococcus lactis and is required for natural protein processing. Mol. Microbiol. 35:1042-1051. https://doi.org/10.1046/j.1365-2958 .2000.01757.x.
Qi, W., Z. C. Fan, C. L. Wang, L. H. Hou, J. F. Liu, and X. H. Cao. 2014. Non-targeted metabolomic reveals the effect of salt stress on global metabolite of halotolerant yeast Candida versatilis and principal component analysis. J. Ind. Microbiol. Biotechnol. 41:1553-1562. https://doi.org/10.1007/s10295-014-1475-7.

Roy, A., R. Dutta, N. Kundu, D. Banik, and N. Sarkar. 2016. A comparative study of the influence of sugars sucrose, trehalose, and maltose on the hydration and diffusion of DMPC lipid bilayer at complete hydration: investigation of structural and spectroscopic aspect of lipid-sugar interaction. Langmuir 32:5124-5134. https:// doi.org/10.1021/acs.langmuir.6b01115.

Ruggirello, M., M. Giordano, M. Bertolino, I. Ferrocino, L. Cocolin, and P. Dolci. 2018. Study of Lactococcus lactis during advanced ripening stages of model cheeses characterized by GC-MS. Food Microbiol. 74:132-142. https://doi.org/10.1016/j.fm.2018.03.012.

Sanders, J. W., G. Venema, and J. Kok. 1999. Environmental stress responses in Lactococcus lactis. FEMS Microbiol. Rev. 23:483-501. https://doi.org/10.1111/j.1574-6976.1999.tb00409.x.

Sauer, M., H. Russmayer, R. Grabherr, C. K. Peterbauer, and H. Marx. 2017. The efficient clade: Lactic acid bacteria for industrial chemical production. Trends Biotechnol. 35:756-769. https://doi .org/10.1016/j.tibtech.2017.05.002.

Serrazanetti, D. I., M. E. Guerzoni, A. Corsetti, and R. Vogel. 2009. Metabolic impact and potential exploitation of the stress reactions in lactobacilli. Food Microbiol. 26:700-711. https://doi.org/ 10.1016/j.fm.2009.07.007.

Sharma, V., M. Ichikawa, and H. H. Freeze. 2014. Mannose metabolism: More than meets the eye. Biochem. Biophys. Res. Commun. 453:220-228. https://doi.org/10.1016/j.bbrc.2014.06.021.

Sheng, Y., X. Yang, Y. Lian, B. Zhang, X. He, W. Xu, and K. Huang. 2016. Characterization of a cadmium resistance Lactococcus lactis subsp. lactis strain by antioxidant assays and proteome profiles methods. Environ. Toxicol. Pharmacol. 46:286-291. https://doi .org/10.1016/j.etap.2016.08.008.

Shenoy, N., E. Creagan, T. Witzig, and M. Levine. 2018. Ascorbic acid in cancer treatment: Let the phoenix fly. Cancer Cell 34:700-706. https://doi.org/10.1016/j.ccell.2018.07.014.

Shin, H., S.-J. Hong, H. Kim, C. Yoo, H. Lee, H.-K. Choi, C.-G. Lee, and B.-K. Cho. 2015. Elucidation of the growth delimitation of Dunaliella tertiolecta under nitrogen stress by integrating transcriptome and peptidome analysis. Bioresour. Technol. 194:57-66. https://doi.org/10.1016/j.biortech.2015.07.002.

Shiomi, N., and S. Onodera. 1988. BETA-Configuration of D-fructofuranose released by the action of invertases on sucrose. Agric. Biol. Chem. 52:2347-2348. https://doi.org/10.1271/bbb1961.52.2347.

Singh, N., R. R. Kuppili, and K. Bose. 2011. The structural basis of mode of activation and functional diversity: A case study with HtrA family of serine proteases. Arch. Biochem. Biophys. 516:8596. https://doi.org/10.1016/j.abb.2011.10.007.

Termont, S., K. Vandenbroucke, D. Iserentant, S. Neirynck, L. Steidler, E. Remaut, and P. Rottiers. 2006. Intracellular accumulation of trehalose protects Lactococcus lactis from freeze-drying damage and bile toxicity and increases gastric acid resistance. Appl. Environ. Microbiol. 72:7694-7700. https://doi.org/10.1128/AEM $.01388-06$.

Tian, K., Y. Li, B. Wang, H. Wu, Q. Caiyin, Z. Zhang, and J. Qiao. 2019. The genome and transcriptome of Lactococcus lactis ssp. lactis F44 and G423: Insights into adaptation to the acidic environment. J. Dairy Sci. 102:1044-1058. https://doi.org/10.3168/jds .2018-14882.

Torres Manno, M., F. Zuljan, S. Alarcón, L. Esteban, V. Blancato, M. Espariz, and C. Magni. 2018. Genetic and phenotypic features defining industrial relevant Lactococcus lactis, L. cremoris and $L$. lactis biovar. diacetylactis strains. J. Biotechnol. 282:25-31. https: //doi.org/10.1016/j.jbiotec.2018.06.345.

van de Guchte, M., P. Serror, C. Chervaux, T. Smokvina, S. D. Ehrlich, and E. Maguin. 2002. Stress responses in lactic acid bacteria. Antonie van Leeuwenhoek 82:187-216. https://doi.org/10.1023/A 1020631532202.

van Hylckama Vlieg, J. E. T., J. L. W. Rademaker, H. Bachmann, D. Molenaar, W. J. Kelly, and R. J. Siezen. 2006. Natural diversity 
and adaptive responses of Lactococcus lactis. Curr. Opin. Biotechnol. 17:183-190. https://doi.org/10.1016/j.copbio.2006.02.007.

Vismeh, R., D. Haddad, J. Moore, C. Nielson, B. Bals, T. Campbell, A. Julian, F. Teymouri, A. D. Jones, and V. Bringi. 2018. Exposure assessment of acetamide in milk, beef, and coffee using xanthydrol derivatization and gas chromatography/mass spectrometry. J. Agric. Food Chem. 66:298-305. https://doi.org/10.1021/ acs.jafc.7b02229.

Wu, H., Y. Zhao, Y. Du, S. Miao, J. Liu, Y. Li, Q. Caiyin, and J. Qiao. 2018. Quantitative proteomics of Lactococcus lactis F44 under cross-stress of low pH and lactate. J. Dairy Sci. 101:6872-6884. https://doi.org/10.3168/jds.2018-14594.

Yang, F., X. Lu, H. Zong, H. Ji, and B. Zhuge. 2018. Gene expression profiles of Candida glycerinogenes under combined heat and highglucose stresses. J. Biosci. Bioeng. 126:464-469. https://doi.org/10 .1016/j.jbiosc.2018.04.006.

Yeoman, C. J., Y. Han, D. Dodd, C. M. Schroeder, R. I. Mackie, and I. K. O. Cann. 2010. Chapter 1 - Thermostable enzymes as biocatalysts in the biofuel industry. Pages 1-55 in Advances in Applied Microbiology. Vol. 70. Academic Press, London, UK.

Yerlikaya, O. 2019. Probiotic potential and biochemical and technological properties of Lactococcus lactis ssp. lactis strains isolated from raw milk and kefir grains. J. Dairy Sci. 102:124-134. https:/ /doi.org/10.3168/jds.2018-14983.

Yu, J., W. Hui, C. Cao, L. Pan, H. Zhang, and W. Zhang. 2018. Integrative genomic and proteomic analysis of the response of Lactobacillus casei Zhang to glucose restriction. J. Proteome Res. 17:1290-1299. https://doi.org/10.1021/acs.jproteome.7b00886.

Yu, J.-L., X.-X. Xia, J.-J. Zhong, and Z.-G. Qian. 2017. A novel synthetic pathway for glutarate production in recombinant Escherich- ia coli. Process Biochem. 59:167-171. https://doi.org/10.1016/j procbio.2017.06.026.

Zarzecka, U., A. Modrak-Wojcik, M. Bayassi, M. Szewczyk, A. Gieldon, A. Lesner, T. Koper, A. Bzowska, M. Sanguinetti, S. Backert, B. Lipinska, and J. Skorko-Glonek. 2018. Biochemical properties of the HtrA homolog from bacterium Stenotrophomonas maltophilia. Int. J. Biol. Macromol. 109:992-1005. https://doi.org/10.1016/j .ijbiomac.2017.11.086.

Zheng, F., W. Zhang, Y. Sui, R. Ding, W. Yi, Y. Hu, H. Liu, and C. Zhu. 2019. Sugar protectants improve the thermotolerance and biocontrol efficacy of the biocontrol yeast, Candida oleophila. Front. Microbiol. 10:187. https://doi.org/10.3389/fmicb.2019.00187.

Zhu, Z. M., P. S. Yang, Z. M. Wu, J. Zhang, and G. C. Du. 2019. Systemic understanding of Lactococcus lactis response to acid stress using transcriptomics approaches. J. Ind. Microbiol. Biotechnol. 46:1621-1629. https://doi.org/10.1007/s10295-019-02226-x.

Zuljan, F. A., P. Mortera, S. H. Alarcón, V. S. Blancato, M. Espariz, and C. Magni. 2016. Lactic acid bacteria decarboxylation reactions in cheese. Int. Dairy J. 62:53-62. https://doi.org/10.1016/j.idairyj .2016.07.007.

\section{ORCIDS}

Wei Qi ® https://orcid.org/0000-0001-7977-7792

Xiao-Xue Li 이 https://orcid.org/0000-0002-1180-4181

Yao-Hua Guo @ https://orcid.org/0000-0001-8897-8123

Yan-Zhou Bao @ https://orcid.org/0000-0002-3156-5720

Nan Wang ๑ https://orcid.org/0000-0003-4640-9090 